MATHEMATICS OF COMPUTATION

Volume 70, Number 233 , Pages $223-250$

S $0025-5718(00) 01261-8$

Article electronically published on June 12, 2000

\title{
TRANSFORMATION OF HYPERSINGULAR INTEGRALS AND BLACK-BOX CUBATURE
}

\author{
S. A. SAUTER AND C. LAGE
}

\begin{abstract}
In this paper, we will consider hypersingular integrals as they arise by transforming elliptic boundary value problems into boundary integral equations. First, local representations of these integrals will be derived. These representations contain so-called finite-part integrals. In the second step, these integrals are reformulated as improper integrals. We will show that these integrals can be treated by cubature methods for weakly singular integrals as they exist in the literature.
\end{abstract}

\section{INTRODUCTION}

In this paper, we will consider Fredholm integral equations on two-dimensional surfaces in $\mathbb{R}^{3}$ which typically arise by applying the boundary element method to boundary value problems (see, e.g., [8], 28]). Such boundary integral equations can be solved numerically by Petrov-Galerkin methods by employing finite dimensional test and trial spaces on the surface. In most practical applications, the surface is piecewise smooth but contains corners and edges. Surface grids consisting of (curved) triangles and quadrilaterals are used to set up these spaces as, e.g., finite element spaces, wavelets, spectral elements, etc. Since finite element spaces are applicable to a broad class of integral equations and flexible to resolve the possibly singular behaviour of a solution by adaptivity, we restrict our consideration to the boundary element method, i.e., finite elements lifted onto surfaces by local charts. Alternative discretisations as, e.g., spectral elements, might be preferable for special situations while they are limited to a comparatively small class of problems (e.g., integral equations on tori and spheres).

From the theoretical point of view, the Galerkin method, where test and trial spaces coincide, is the method of choice since stability and optimal convergence rates can be proved for much more general situations as, e.g., for the so-called collocation method (point matching) and the Nyström method.

With increasing interest in the numerical solution of the Galerkin boundary element method, the need of appropriate cubature 1 methods for computing the elements of the system matrix arises.

For weakly or Cauchy singular integrals, there exist appropriate cubature methods for approximating the elements of the system matrix (see [2, 26], 23, [9], 27,

Received by the editor January 8, 1998.

2000 Mathematics Subject Classification. Primary 65N38, 65R10, 65R20.

Key words and phrases. Finite-part integrals, regularisation, numerical integration, boundary element methods.

${ }^{1}$ In more than one dimension the term quadrature is replaced by cubature. 
[4], 15], [17]). For many important problems as, e.g., mixed boundary value problems or transmission problems, the kernel functions are not integrable in the sense of Cauchy principal values. They are hypersingular and have to be regularised in the sense of Hadamard (see [8], [25]). For these kinds of integrals, cubature methods for Galerkin discretisations are missing in the literature. To overcome this difficulty a regularisation on the continuous level is often applied rendering the integrals weakly or Cauchy singular (see [20, [11, 8]). The drawback of this technique is that it has to be worked out for each kernel function separately, i.e., is not fully implicit. Here and in the following, the term fully implicit is used in the sense that the definition of the cubature method does not depend on the explicit form of the integrand but works as a black-box method for all kernel functions specified in Section 3. Only the subroutine for evaluating the kernel function in pairs of cubature points has to be exchanged.

In our paper we present a direct approach for evaluating hypersingular integrals which are efficient in the sense that this family of cubature rules is

1. fully implicit,

2. exponentially convergent (with respect to the order of the rule),

3. uniformly stable (with respect to the order of the rule).

The main purpose of this paper is the development of transformation techniques rendering the integrands analytic. Gauß-Legendre cubature rules applied to these integrands will converge exponentially with respect to the order. The development of such regularising transformations requires a careful analysis of various singularities occurring when applying hypersingular Fredholm integral operators to finite element spaces on surfaces. The relevant properties will be derived in the first part of the paper. The quantitative estimates of the local cubature errors and their influence to the global discretisation error will be the topic of a forthcoming paper which will also contain numerical experiments. The general theory of fully discrete Galerkin methods including numerical cubature has been worked out in [5] and 24]. The orders of integration for weakly singular and Cauchy singular kernel can be found in [4].

For collocation methods, such techniques are described in [7, [6], 26], [13. We will use these results to analyse the behaviour of the integrand for the outer integration appearing for the Galerkin method. For the special situation of 1) piecewise flat surfaces and 2) the hypersingular kernel function corresponding to the Laplace operator, semi-analytic cubature techniques for the Galerkin method are worked out in [14].

The paper is organised as follows. In Section 2 we will specify the class of boundary integral equations which will be considered and formulate the Galerkin discretisation of the arising weak formulation.

In Section 3 properties of boundary integral equations and corresponding kernel functions are collected.

Then in Section 4 it is explained how the arising finite-part integrals (over the whole surface) can be localized as finite-part integrals over pairs of panels.

In Section 5 the local finite-part integrals are reformulated as a sum of weakly singular integrals by analysing the singular behaviour of the arising integrands.

Finally, in Section 6 families of cubature rules are defined for the approximation of the derived weakly singular integrals which converge exponentially with respect to the order. 
In this paper, we will consider the boundary element method based on a partitioning of the surface into (curved) triangles. However, the theoretical results apply also for meshes consisting of quadrilaterals as well as for meshes containing both triangles and quadrilaterals. The coordinate transforms for such general meshes have the same structure but, obviously, a different form. For readers interested in these cases, we refer to the extended version of this paper [22] available via the internet address http://www.numerik.uni-kiel.de/reports/1997/.

\section{The BOUNDARY ELEMENT METHOD}

Let $\Gamma$ be a piecewise analytic, orientable surface of a bounded Lipschitz domain $\Omega \subset \mathbb{R}^{3}$ (see [21, p. 3]). The assumption on the analyticity of $\Gamma$ is merely imposed for convenience. We expect that this condition can be replaced by "sufficiently smooth" in a similar fashion as worked out in [19. However, the detailed extension of the theory below to that more general case is not worked out yet.

Let $L^{2}(\Gamma)$ denote the space of all measurable functions $u: \Gamma \rightarrow \mathbb{C}$ which are square integrable with respect to the surface measure $d \Gamma . H^{1}(\Gamma)$ is defined as usual by employing a Lipschitz atlas and a partition of unity. The intermediate spaces $H^{s}(\Gamma), 0<s<1$, are defined via interpolation while, for $-1 \leq s<0, H^{s}(\Gamma)$ is the dual space of $H^{-s}(\Gamma)$ with respect to the $L^{2}$-scalar product. This scalar product in $L^{2}(\Gamma)$ can be extended continuously to $H^{s}(\Gamma) \times H^{-s}(\Gamma)$ and is denoted by $(\cdot, \cdot)_{0}$.

For $i=1,2$, consider the operators

$$
\lambda_{i} I+\mathcal{K}_{i}: H^{s_{i}} \rightarrow H^{-s_{i}},
$$

where $s_{1}, s_{2} \in[-1,1]$ and the functions $\lambda_{1}, \lambda_{2}$ are analytic on smooth parts of the surface. The integral operators $\mathcal{K}_{i}, i=1,2$, are given by

$$
\mathcal{K}_{i}[w](x)=p \cdot f \cdot \int_{\Gamma} k_{i}(x, y, y-x) w(y) d \Gamma_{y} .
$$

If the kernel function contains nonintegrable singularities, then the integral (2.2) has to be understood in the regularised sense of Hadamard, which will be explained in subsection 3.2.

We assume that

- for $\sigma \in\left\{0, s_{2}-s_{1}\right\}$, the operators

$$
\begin{array}{ll}
\lambda_{1} I+K_{1} & : \quad H^{s_{1}} \rightarrow H^{-s_{1}}, \\
\lambda_{2} I+K_{2} & : \quad H^{s_{2}+\sigma} \rightarrow H^{-s_{2}+\sigma}
\end{array}
$$

are continuous.

- the operator $\lambda_{1} I+\mathcal{K}_{1}$ satisfies a Gårding inequality, i.e., there exist $\varepsilon>0$ and constants $c_{1}, c_{2}$ such that

$$
\left(u,\left(\lambda_{1} I+\mathcal{K}_{1}\right) u\right)_{0} \geq c_{1}\|u\|_{s_{1}}^{2}-c_{2}\|u\|_{s_{1}-\varepsilon}^{2}
$$

is satisfied for all $u \in H^{s_{1}}(\Gamma)$.

We consider Fredholm integral equations in the variational form. For given

$$
f \in H^{2 s_{2}-s_{1}}(\Gamma),
$$

we are seeking $u \in H^{s_{1}}(\Gamma)$ such that

$$
\left(v, \lambda_{1} u\right)_{0}+\left(v, \mathcal{K}_{1} u\right)_{0}=\left(v, \lambda_{2} f\right)_{0}+\left(v, \mathcal{K}_{2} f\right)_{0}, \quad \forall v \in H^{s_{1}}(\Gamma) .
$$


The left-hand side of (2.6) defines the bilinear form $a: H^{s_{1}}(\Gamma) \times H^{s_{1}}(\Gamma) \rightarrow \mathbb{C}$ and the right-hand side the functional $F \in H^{-s_{1}}(\Gamma)$. In compact form, the variational problem is given by seeking $u \in H^{s_{1}}(\Gamma)$ so that

$$
a(u, v)=F(v), \quad \forall v \in H^{s_{1}}(\Gamma)
$$

is satisfied. Throughout this paper we assume that

$$
s_{1}, s_{2} \in\left\{-\frac{1}{2}, 0, \frac{1}{2}\right\}
$$

holds (this requirement is satisfied for most practical applications in three dimensions). However, we hasten to say that our theory is by no means limited to this case and can be generalised to more general integral operators (see Remark 4 ).

The Galerkin discretisation of (2.6) is given by replacing the Sobolev space $H^{s_{1}}(\Gamma)$ by a finite dimensional subspace which will be constructed below.

Let $\tilde{\Gamma}$ be the (piecewise plane) surface of a polyhedron which interpolates $\Gamma$. Let $\tilde{\tau}:=\left\{\tilde{K}_{1}, \tilde{K}_{2}, \ldots, \tilde{K}_{N}\right\}$ denote a grid on the surface $\tilde{\Gamma}$ consisting of plane (open) triangles satisfying

$$
\begin{aligned}
& \tilde{\Gamma}=\widetilde{\bigcup_{\tilde{K} \in \tilde{\tau}} \tilde{K}} \\
& \widetilde{K} \cap \widetilde{K^{\prime}}=\emptyset, \quad \forall \widetilde{K}, \widetilde{K^{\prime}} \in \tilde{\tau} \text { with } \widetilde{K} \neq \widetilde{K^{\prime}} .
\end{aligned}
$$

The following assumption links the true surface $\Gamma$ with the auxiliary surface $\tilde{\Gamma}$. We assume that there exists a bi-Lipschitz mapping $\eta: \tilde{\Gamma} \rightarrow \Gamma$ having the property that, for all $\widetilde{K} \in \tilde{\tau}$, the restriction $\left.\eta\right|_{\tilde{K}}$ can be extended to an analytic mapping $\eta: \bar{K} \rightarrow \Gamma$ and the inverse $\eta^{-1}$ has the analogue property.

The grid $\tilde{\tau}$ induces a grid on the true surface $\Gamma$ by

$$
\tau:=\{\eta(\tilde{K}): \tilde{K} \in \tilde{\tau}\}
$$

The space of finite element functions on the surface $\Gamma$ is defined as usual by lifting polynomial spaces on a reference element onto the true surface. Let $P_{K}$ denote the space of bivariate polynomials of total degree $p$. The reference triangle is given by

$$
Q:=\operatorname{int} \operatorname{conv}\left((0,0)^{T},(1,0)^{T},(0,1)^{T}\right),
$$

where "conv" denotes the convex hull and "int" the interior of a set. The triangle $Q$ is mapped onto a surface triangle $K$ by a composition of $\eta$ with an affine mapping. For $K \in \tau$, let $\widetilde{K}:=\eta^{-1}(K)$. The affine mapping $\kappa_{K}: Q \rightarrow \widetilde{K}$ is given by:

$$
\kappa_{K}(\hat{x})=A+(B-A) \hat{x}_{1}+(C-A) \hat{x}_{2},
$$

where $A, B, C$ denote the vertices of $\tilde{K}$ (counterclockwise ordering). We emphasize that, throughout the paper, all triangles are open sets.

For $r \in \mathbb{N}_{0}$, the finite element space $S_{\tau}^{r, p}$ is defined by

$$
S_{\tau}^{r, p}:=\left\{u \in C^{r}(\Gamma)|\forall K \in \tau: u|_{K} \circ \eta \circ \kappa_{K} \in P_{K}\right\} .
$$

For $r=-1$, the condition $u \in C^{r}(\Gamma)$ in (2.8) has to be replaced by $u \in L^{\infty}(\Gamma)$. For continuous finite elements, i.e., $r \geq 0$, we assume that, for all $K, K^{\prime} \in \tau, K^{\prime} \neq K$, the intersection $\bar{K} \cap \overline{K^{\prime}}$ is either empty, a common vertex, or a common edge. In the following, we write $V_{\tau}$ short for $S_{\tau}^{r, p}$. 
The Galerkin discretisation of (2.6) is given by finding $u_{G} \in V_{\tau}$ such that

$$
a\left(u_{G}, v\right)=F(v), \quad \forall v \in V_{\tau} .
$$

This problem can be reformulated as a system of linear equations by introducing the basis representation of $u_{G}$ :

$$
u_{G}(x)=\sum_{i=1}^{n} \mathbf{u}_{i} \varphi_{i}(x),
$$

where $n:=\operatorname{dim} V_{\tau}$. Then, (2.9) is equivalent to

$$
\mathbf{A u}=\mathbf{F},
$$

where the system matrix $\mathbf{A} \in \mathbb{C}^{n \times n}$ and the vector $\mathbf{F} \in \mathbb{C}^{n}$ are given by

$$
\begin{aligned}
\mathbf{A}_{i, j} & =a\left(\varphi_{i}, \varphi_{j}\right), \\
\mathbf{F}_{i} & =F\left(\varphi_{i}\right) .
\end{aligned}
$$

To compute the matrix entries $\mathbf{A}$ and the right-hand side $\mathbf{F}$ fast cubature techniques are needed for the evaluation of

$$
\begin{aligned}
& \int_{\Gamma} \varphi_{i}(x) \varphi_{j}(x) \lambda(x) d x, \\
& \int_{\Gamma} \varphi_{i}(x) p . f . \int_{\Gamma} k(x, y, y-x) \varphi_{j}(y) d \Gamma_{y} d \Gamma_{x},
\end{aligned}
$$

where $\lambda$ is analytic on smooth parts of the surface and the kernel function $k$ is either $k_{1}$ or $k_{2}$. The evaluation of the first integral is not problematic, and we will discuss in the following only the second one. In this paper, we will focus on the definition of cubature rules for the numerical integration of (2.10) which approximate (2.10) to any required accuracy with a priori known convergence behaviour. Quantitative estimates of the local cubature error and the effect of replacing the true Galerkin matrix by a cubature approximation on the global discretisation error is studied thoroughly for weakly and Cauchy singular kernel in [24, 4], [5]. The extension to hypersingular operators and formulae for the required cubature orders will be the topic of a forthcoming paper.

\section{Properties of boundary integral equations}

3.1. The kernel function. The properties of an integral operator

$$
\mathcal{K}[u](x)=p . f . \int_{\Gamma} k(x, y, y-x) u(y) d \Gamma_{y}
$$

are determined by the kernel function $k: \Gamma \times \Gamma \rightarrow \mathbb{C}$. We assume that $k$ has the following representation

$$
k(x, y, z)=\|z\|^{-s} \sum_{i, j=0}^{b} \kappa_{i}(x) \rho_{j}(y) A_{i, j}\left(\|z\|, \frac{z}{\|z\|}\right), \quad \forall x, y \in \Gamma, z=y-x, x \neq y,
$$

where $b$ is a finite number, and $A_{i, j}(r, \xi)$ is analytic with respect to $r$ in any compact neighbourhood of zero and analytic with respect to $\xi$ in a neighbourhood of the sphere $S_{2}$. The functions $\kappa_{i}, \rho_{i}$ are assumed to be in $L^{\infty}(\Gamma)$ and analytic on analytic parts of the boundary. To be more precise, we assume that, for all $K \in \tau$, the restrictions $\left.\kappa_{i}\right|_{K}$ and $\left.\rho_{i}\right|_{K}$ are analytic. We state that practically 
all kernel functions arising by transforming elliptic boundary value problems into integral equations are of the form (3.2) (see [5] and the references therein). The kernel functions are associated with fundamental solutions to differential equations. The following examples illustrate that the fundamental solution of elliptic, scalar differential operators in $\mathbb{R}^{3}$ are of the form (3.2).

Example 1. Let $G \in \mathbb{R}^{3 \times 3}$ be a symmetric and positive definite matrix, let $\beta \in \mathbb{R}^{3}$ and $c \in \mathbb{R}$. Consider the differential operator

$$
L u=-\operatorname{div}(G \operatorname{grad} u)+2\langle\beta, \operatorname{grad} u\rangle+c u .
$$

Put $\langle\cdot, \cdot\rangle_{G}=\left\langle G^{-1} \cdot, \cdot\right\rangle$ and $\|\cdot\|_{G}=\langle\cdot, \cdot\rangle_{G}^{1 / 2}$. The fundamental solution of $L$ (satisfying $L S=\delta_{0}$ with $\delta_{0}$ denoting Dirac's functional centred at the origin) is given by

$$
S(z)=\frac{1}{4 \pi \sqrt{\operatorname{det} G}} \frac{e^{\langle\beta, z\rangle_{G}-\lambda\|z\|_{G}}}{\|z\|_{G}}
$$

with $\lambda^{2}=c+\|\beta\|_{G}^{2}$. This function can be rewritten as

$$
\|z\|^{-1} A\left(\|z\|, \frac{z}{\|z\|}\right)
$$

with the function $A$ defined by

$$
A(r, \xi)=\frac{1}{4 \pi \sqrt{\operatorname{det} G}} \frac{e^{r\left\{\langle\beta, \xi\rangle_{G}-\lambda\|\xi\|_{G}\right\}}}{\|\xi\|_{G}} .
$$

Hence, $S(z)$ is of the form (3.2) and satisfies the analyticity properties due to the regularity of $G$.

Example 2. The kernel of the classical double layer potential for Laplace's equation in $3 \mathrm{D}$ is the normal derivative of (3.3) with $G=I, \beta=0, c=0$ :

$$
k(x, y, z)=-\frac{\langle n(y), z\rangle}{4 \pi\|z\|^{3}} .
$$

This function can be rewritten as

$$
k(x, y, z)=-\|z\|^{-2} \sum_{i=1}^{3}\left(\frac{n_{i}(y)}{4 \pi}\right) \frac{z_{i}}{\|z\|} .
$$

Since the components of the normal vector $n$ are piecewise analytic, the kernel function is of the form (3.2).

Finally, we remark that the kernel functions arising from the Lamé equation and the velocity part of kernel functions corresponding to the Stokes equation satisfy our general assumptions on $k$ too.

In our paper, we will concentrate on elliptic boundary value problems of second order. In [5] it is explained that for such problems the order of singularity $s$ in (3.2) typically satisfies

$$
s \leq 3
$$

For $s \leq 2$ (in combination with the so-called Giraud-Mikhlin condition, see [25] formula (11)] and [18, Chap. 9]), the finite-part integral reduces to a Cauchy principal value where transformation rules and cubature techniques already exist in the 
literature (see [8], [23], 9], [27], 4]). In this paper, we will assume throughout that

$$
s=3
$$

holds. We state that all our statements remain valid also for $s \leq 3$, while some of the assumptions can be weakened and formulae simplified. In Remark 4, it is explained how our results can be extended to the case $s>3$.

3.2. Finite-part integrals. We come now to the definition of the finite part integral involved in (3.1). For this, let $x \in \Gamma$ be a point inside a smooth part of the surface and, for $\varepsilon>0$, let $B_{\varepsilon}(x)$ denote the (three-dimensional) ball with radius $\varepsilon$ centred at $x$. Let $\gamma \subset \Gamma$ be a measurable subset of $\Gamma$ satisfying $x \notin \partial \gamma$. We consider a function $u \in L^{\infty}(\gamma)$ being smooth in a neighbourhood of $x$ (Hölder continuous with exponent $\lambda>1$ is sufficient). Since the kernel function $k(x, y, y-x)$ is bounded for $y \neq x$ the following integral exists as a usual Riemann integral

$$
I_{\varepsilon, \gamma}[u](x):=\int_{\gamma \backslash B_{\varepsilon}(x)} k(x, y, y-x) u(y) d \Gamma_{y} .
$$

In [25] and [12], it was shown that the functional $I_{\varepsilon, \gamma}$ admits an expansion as

$$
I_{\varepsilon, \gamma}[u](x)=A_{-1, \gamma}[u](x) \varepsilon^{-1}+A_{\log , \gamma}[u](x) \log \varepsilon+A_{0, \gamma}[u](x)+R_{\varepsilon, \gamma}[u](x),
$$

where $R_{\varepsilon, \gamma}[u](x) \rightarrow 0$ as $\varepsilon \rightarrow 0$. The finite part integral then is defined by

$$
p . f . \int_{\gamma} k(x, y, y-x) u(y) d \Gamma_{y}:=A_{0, \gamma}[u](x) .
$$

In [25] and [12], it was proved that the right-hand side above is finite.

The following general assumption on the integral operator $\mathcal{K}$ in (3.1) is assumed throughout the paper. $\mathcal{K}$ is a bounded operator from $H^{\mu}$ onto $H^{-\mu}$ with $\mu \in$ $\left\{-\frac{1}{2}, 0, \frac{1}{2}\right\}$. For computation of the matrix elements, the integrals

$$
\left(\varphi_{q}, \mathcal{K}\left[\varphi_{r}\right]\right)_{0}, \quad 1 \leq q, r \leq n,
$$

have to be evaluated. For an arbitrary function $v \in H^{\mu}$, the image $\mathcal{K}[v]$ lies in $H^{-\mu}$ and, for $\mu=1 / 2$, does not belong necessarily to $L^{2}$. This would complicate the development of cubature techniques for approximating the dual pairing $(w, \mathcal{K}[v])_{0}=(w, \mathcal{K}[v])_{\mu \times-\mu}$ substantially. In particular, the splitting

$$
(w, \mathcal{K}[v])_{0}=\int_{\Gamma} w \mathcal{K}[v] d x=\sum_{K \in \tau} \int_{K} w \mathcal{K}[v] d x=\sum_{K \in \tau}(w, \mathcal{K}[v])_{L^{2}(K)}
$$

is not valid for all functions $v, w \in H^{\mu}(\Gamma)$. However, in many cases the operator $\mathcal{K}$ satisfies a so-called shift property, i.e., $\mathcal{K}$ is a bounded operator from $H^{\mu+\sigma}$ into $H^{-\mu+\sigma}$ for a certain range of $\sigma$. For our purposes, it is sufficient to assume throughout the paper that

$$
\begin{aligned}
& \mathcal{K}: \quad H^{\mu}(\Gamma) \rightarrow H^{-\mu}(\Gamma), \\
& \mathcal{K}: \quad H^{1}(\Gamma) \rightarrow L^{2}(\Gamma)
\end{aligned}
$$

is bounded.

Corollary 1. Let (3.6) and (3.7) be satisfied. The definition (2.8) of the finitedimensional spaces $V_{\tau}$ and $V_{\tau} \subset H^{\mu}$ implies that

$$
\mathcal{K}[u] \in L^{2}(\Gamma), \quad \forall u \in V_{\tau} .
$$


Proof. For $\mu \leq 0$, the assertion follows from (3.6) and $V_{\tau} \subset H^{\mu}$ via $\mathcal{K}\left[V_{\tau}\right] \subset H^{-\mu} \subset$ $L^{2}(\Gamma)$. For $\mu=1 / 2$, all functions in $V_{\tau}$ are Lipschitz continuous and the result follows from (3.7) by using $V_{\tau} \subset C^{0,1}(\Gamma) \subset H^{1}(\Gamma)$.

A comment on the validity of (3.7) is given below.

Remark 1. Assumption (3.7) is satisfied, e.g., for the hypersingular integral operators corresponding to elliptic boundary value problems of second order with the Laplace operator as the principal part, discretised by $S_{\tau}^{r, p}$ for $r \geq 0$ (for a proof, see [3]).

\section{LOCAL REPRESENTATION OF HYPERSINGULAR INTEGRALS}

For the approximation of the integrals (3.5), it is important to localize the integrals over the whole surface $\Gamma$ by splitting them into a sum over the panels and to transform these local integrals onto fixed reference panels. Then, it suffices to develop cubature rules on these reference elements. In view of the finite part integrals, this splitting and transformation is much more delicate as for weakly singular integrals where such transformations are straightforward. For simplicity, we abbreviate the integrand in 3.5 with

$$
k^{\text {new }}(x, y, z):=\varphi_{q}(x) \varphi_{r}(y) k(x, y, z)
$$

and skip the superscript new in the following. For $K_{i}, K_{j} \in \tau$, we define the function $H_{i, j}: K_{i} \rightarrow \mathbb{C}$ by

$$
H_{i, j}(x):=p . f . \int_{K_{j}} k(x, y, y-x) d \Gamma_{y} \quad \forall x \in K_{i} .
$$

For $t \in\{i, j\}$, let $\eta_{t}:=\eta \circ \kappa_{K_{t}}$ (see (2.7)). For the following it is important that the reference triangle $Q$ (like the surface elements $K_{t}$ ) is assumed to be open.

The local kernel function is defined by

$$
k_{i, j}(\hat{x}, \hat{y}):=k\left(\eta_{i}(\hat{x}), \eta_{j}(\hat{y}), \eta_{j}(\hat{y})-\eta_{i}(\hat{x})\right) g_{i}(\hat{x}) g_{j}(\hat{y}),
$$

where, for $t \in\{i, j\}$, the function $g_{t}$ denotes the surface area element corresponding to the chart $\eta_{t}$. The local version of $H_{i, j}$ defines a mapping $\hat{H}_{i, j}: Q \rightarrow \mathbb{C}$ by

$$
\hat{H}_{i, j}(\hat{x}):=p . f \cdot \int_{Q} k_{i, j}(\hat{x}, \hat{y}) d \hat{y} \quad \forall \hat{x} \in Q .
$$

Note that, for the regularisation of the finite-part integral in (4.1), an $\varepsilon$-ball in the parameter plane has to be subtracted. It is not necessary to perform the limit with respect to the distorted ball $\eta_{i}^{-1}\left(B_{\varepsilon}(x) \cap K_{i}\right)$. This fact will simplify the treatment of the hypersingular integrals substantially.

The connection of $H_{i, j}$ and $\hat{H}_{i, j}$ is expressed by the formula

$$
g_{i}(\hat{x})\left(H_{i, j} \circ \eta_{i}\right)(\hat{x})=\hat{H}_{i, j}(\hat{x}) \quad \forall \hat{x} \in Q,
$$

which is proved in [25, Theorem 5]. The sum

$$
\sum_{K_{j} \in \tau} \hat{H}_{i, j}(\hat{x})=: \hat{H}_{i}(\hat{x}) \quad \forall \hat{x} \in Q
$$

can be regarded as a local version of the integral operator $\mathcal{K}$ (up to a bounded factor):

$$
g_{i}(\hat{x}) \varphi_{q}(x) \mathcal{K}\left[\varphi_{r}\right](x)=\hat{H}_{i}(\hat{x}) \quad \forall \hat{x} \in Q, \quad x=\eta_{i}(\hat{x}) .
$$


The mapping property of $\mathcal{K}$ (see [3.8) implies $\hat{H}_{i} \in L^{2}(\Gamma)$. It follows that the integral (3.5) equals

$$
\sum_{K_{i} \in \tau} \int_{Q} \sum_{K_{j} \in \tau} \hat{H}_{i, j}(\hat{x}) d \hat{x}
$$

In Lemma 8 , we will prove that $\hat{H}_{i, j}$ is possibly singular only if $\hat{x} \rightarrow \partial Q$. In this light, we define, for $\delta>0$, the reduced element $Q^{\delta}$ by

$$
Q^{\delta}:=\left\{\begin{array}{l}
\delta<\hat{x}_{1}<1-2 \delta \\
\delta<\hat{x}_{2}<1-\delta-\hat{x}_{1}
\end{array}\right\}
$$

It follows from the weak singularity of $\hat{H}_{i}(\hat{x})$ that

$$
\int_{Q} \hat{H}_{i}(\hat{x}) d \hat{x}=\lim _{\delta \rightarrow 0} \int_{Q^{\delta}} \sum_{K_{j} \in \tau} \hat{H}_{i, j}(\hat{x}) d \hat{x}<\infty .
$$

Since $\hat{H}_{i, j}$ is possibly singular only if $\hat{x} \rightarrow \partial Q$ (see Lemma 8 ), the integrand is bounded on $Q^{\delta}$ and we may interchange the summation with the integration

$$
\int_{Q} \hat{H}_{i}(\hat{x}) d \hat{x}=\lim _{\delta \rightarrow 0} \sum_{K_{j} \in \tau} \int_{Q^{\delta}} \hat{H}_{i, j}(\hat{x}) d \hat{x} .
$$

In Lemma 2 (c), Lemma 4 and Lemma 7, we will prove that the integrals on the right-hand side above have an expansion of the form

$$
\int_{Q^{\delta}} \hat{H}_{i, j}(\hat{x}) d \hat{x}=I_{\log }^{i, j} \log \delta+I_{0}^{i, j}+I_{1}^{i, j}(\delta),
$$

where $I_{1}^{i, j}(\delta) \rightarrow 0$ as $\delta \rightarrow 0$. From the boundedness of the integral (4.3), it follows that

$$
\sum_{K_{j} \in \tau} I_{\log }^{i, j}=0
$$

holds. This motivates the definition of a further finite-part integral:

$$
p . f . \int_{Q} \hat{H}_{i, j}(\hat{x}) d \hat{x}:=I_{0}^{i, j} .
$$

Lemma 1. The integral

$$
\int_{\Gamma} p \cdot f \cdot \int_{\Gamma} k(x, y, y-x) d \Gamma_{y} d \Gamma_{x}
$$

has the local representation

$$
\sum_{K_{i} \in \tau} \sum_{K_{j} \in \tau} p \cdot f \cdot \int_{Q} p \cdot f \cdot \int_{Q} k_{i, j}(\hat{x}, \hat{y}) d \hat{y} d \hat{x} .
$$

The inner finite-part integral reduces to the usual Riemann integral if $K_{i} \neq K_{j}$ holds. The outer finite-part integral reduces to an improper integral if $\overline{K_{i}}, \overline{K_{j}}$ share at most one point.

Proof. Let $K_{i} \neq K_{j}$ and $x \in K_{i}$. The pull back $\hat{x}:=\eta_{i}^{-1}(x)$ satisfies $\hat{x} \in Q$. For sufficiently small $\varepsilon>0$, the ball $B_{\varepsilon}(\hat{x})$ has positive distance from $\partial Q$. Hence, the transformed ball $\eta_{i}\left(B_{\varepsilon}(\hat{x})\right)$ has positive distance from $\partial K_{i}$ and, consequently, also positive distance from $K_{j}$. Therefore, the integrand is bounded and the integral converges to the usual Riemann integral as $\varepsilon \rightarrow 0$. 

(c).

If $\overline{K_{i}}$ and $\overline{K_{j}}$ share at most one point, the result follows from Lemma 2 (b),

Remark 2. Formula (4.5) is a local representation of hypersingular kernel functions. The reference element $Q$ is fixed. Thus, it is sufficient to develop cubature rules on $Q$ (see Section 6).

Remark 3. For the special situation of flat triangular panels and the hypersingular kernel function corresponding to the Laplace operator, a splitting of (3.5) into local quantities is derived and worked out in [14]. The approach in the cited paper is different from (4.5); the local quantities are of the form

$$
\underset{\varepsilon}{p . f .} \int_{\substack{K_{i} \\\|x-y\| \geq \varepsilon}} \int_{K_{j}} \ldots d x d y,
$$

while additional line functionals appear in the local representation.

\section{Finite-PART-FREE REPRESENTATION OF HYPERSINGULAR INTEGRALS}

As mentioned before, the single terms $\hat{H}_{i, j}$ are not integrable in general and, hence, the inner sum in (4.2) may not be interchanged with the outer integral. In the following, we will work out the character of the singularity of $\hat{H}_{i, j}$ in detail. These results will play the key role in Section 6 for constructing appropriate variable transforms rendering the integrands analytic such that the integrals can be approximated efficiently by (tensor versions) of Gaussian quadrature rules.

In order to characterise the regularity of the function $\hat{H}_{i, j}$ we will distinguish the following three cases:

I. $\overline{K_{i}}$ and $\overline{K_{j}}$ share at most one common point.

II. $\overline{K_{i}}$ and $\overline{K_{j}}$ share exactly one edge.

III. $K_{i}=K_{j}$.

Case I: From the analyticity of the charts $\eta_{i}, \eta_{j}$ it follows that the functions $g_{i}$, $g_{j}$ and the coefficients $\kappa_{i}, \rho_{j}$ from (3.2) are analytic in local coordinates. The pull backs of the basis functions $\varphi_{q} \circ \eta_{i}, \varphi_{r} \circ \eta_{j}$ are analytic, too. Thus, the singular behaviour of the kernel function is characterized by the singular behaviour of the function $2\|z\|^{-3} A\left(\|z\|, \frac{z}{\|z\|}\right)$ in local coordinates. If $\overline{K_{i}} \cap \overline{K_{j}}=\emptyset$, the kernel function is analytic in local coordinates. Therefore, we assume for the following that the panels share exactly one point: $\overline{K_{i}} \cap \overline{K_{j}}=P$. In local coordinates, the difference $z=y-x$ takes the form

$$
z=\eta_{j}(\hat{y})-\eta_{i}(\hat{x}) .
$$

Without loss of generality we assume that $\eta_{j}\left(\begin{array}{l}0 \\ 0\end{array}\right)=\eta_{i}\left(\begin{array}{l}0 \\ 0\end{array}\right)=P$. Obviously, $z=0$ if and only if $\hat{y}=\hat{x}=(0,0)^{T}$. Taylor expansion of $\eta_{j}$ and $\eta_{i}$ about the origin results in

$$
z=\sum_{m=1}^{\infty} \frac{\left(\langle\hat{y}, \nabla\rangle^{m} \eta_{j}(0)\right)-\left(\langle\hat{x}, \nabla\rangle^{m} \eta_{i}(0)\right)}{m !}
$$

\footnotetext{
${ }^{2}$ For simplicity, we write $A$ instead of $A_{i, j}$.
} 
where the differential operator $\langle\hat{y}, \nabla\rangle^{m}$ is defined by

$$
\langle\hat{y}, \nabla\rangle^{m} \eta_{j}=\sum_{k=0}^{m}\left(\begin{array}{c}
m \\
k
\end{array}\right) \hat{y}_{1}^{k} \hat{y}_{2}^{m-k}\left(\partial_{1}^{k} \partial_{2}^{m-k} \eta_{j}\right) .
$$

Let $\hat{z}=(\hat{x}, \hat{y})$. We introduce four-dimensional polar coordinates by

$$
\hat{z}=r \xi
$$

with $r=\|\hat{z}\|$ and $\xi=\hat{z} /\|\hat{z}\| \in S_{3}$. Then, (5.1) becomes

$$
z=r \sum_{m=0}^{\infty} r^{m} l_{m}(\xi)=: r a_{1}(r, \xi)
$$

with

$$
l_{m}(\xi):=\frac{\left(\left\langle\xi_{34}, \nabla\right\rangle^{m+1} \eta_{j}(0)\right)-\left(\left\langle\xi_{12}, \nabla\right\rangle^{m+1} \eta_{i}(0)\right)}{(m+1) !}
$$

and $\xi_{\theta t}=\left(\xi_{\theta}, \xi_{t}\right)^{T}$. The function $a_{1}(r, \xi)$ is analytic in any compact neighbourhood of $r=0$ and is analytic in $\xi$ in a suitable neighbourhood of $S_{3}$. As in [25, Lemma 1 , Remark 7] one can show that $a_{1}(r, \xi)$ has no zero in a neighbourhood of $r=0$ and $\xi \in S_{3}$. Consequently, $\|z\|^{-s}$ admits the local representation about $r=0$ :

$$
\|z\|^{-s}=r^{-s} a_{2, s}(r, \xi),
$$

where $a_{2, s}$ is analytic in a neighbourhood of $r=0$ and $\xi \in S_{3}$. The ratio $\frac{z}{\|z\|}$ similarly can be expanded by multiplying (5.3) with (5.4) (choosing $s=1$ ) resulting in

$$
\frac{z}{\|z\|}=a_{2,1}(r, \xi) a_{1}(r, \xi)=: a_{3}(r, \xi)
$$

where the function $a_{3}(r, \xi)$ is analytic with respect to $r$ in a neighbourhood of $r=0$ and with respect to $\xi$ in a neighbourhood of $S_{3}$. Combining these expansions we have proven that, for sufficiently small $r$ and $\xi \in S_{3}$, the kernel function $k_{i, j}(x, y)$ can be expressed in local coordinates by

$$
k_{i, j}(\hat{x}, \hat{y})=r^{-s} a_{4}(r, \xi),
$$

where $a_{4}$ is analytic for $r \leq \delta$ with sufficiently small $\delta>0$, and analytic in $\xi$ in a neighbourhood of $S_{3}$. On the other hand, $r>\delta / 2$ implies that $\|x-y\| \geq C \delta$ holds. Hence, in this case the kernel function is analytic, too. It follows that $a_{4}(r, \xi)$ is analytic for all $r=\|\hat{z}\|, \xi=\hat{z} /\|\hat{z}\|$ with $\hat{z}=(\hat{x}, \hat{y})$ and all $\hat{x}, \hat{y} \in \bar{Q}$.

Proposition 1. Let $\overline{K_{i}}$ and $\overline{K_{j}}$ share at most one common point (Case I). Then, the integral

$$
\int_{Q} \int_{Q} k_{i, j}(\hat{x}, \hat{y}) d \hat{y} d \hat{x}
$$

exists as an improper integral.

Proof. Due to the analyticity of $a_{4}$, the integrand can be estimated by $\mathrm{cr}^{-s}$. By introducing four-dimensional polar coordinates $(\hat{x}, \hat{y})=r \psi\left(\alpha_{1}, \alpha_{2}, \alpha_{3}\right)$ with $r^{2}=$ $\left\|\hat{x}^{2}\right\|+\|\hat{y}\|^{2}$ and $\psi=\frac{(\hat{x}, \hat{y})^{T}}{r} \in S_{3}$, the integrand in the new variables can be estimated by the constant $c$ from which the integrability follows. 
Lemma 2. (a) If $\overline{K_{i}}$ and $\overline{K_{j}}$ share at most one point, then the function $\hat{H}_{i, j}$ has the representation

$$
\hat{H}_{i, j}(\hat{x})=\int_{Q} k_{i, j}(\hat{x}, \hat{y}) d \hat{y}
$$

where, for all $\hat{x} \in Q$, the integral exists as a usual Riemann integral.

(b) The function $\hat{H}_{i, j}(\hat{x})$ is weakly singular, i.e., $\int_{Q} \hat{H}_{i, j}(\hat{x}) d \hat{x}$ coincides with (5.6).

(c) Expansion (4.4) is valid with $I_{\log }^{i, j}=0$ :

$$
\int_{Q^{\delta}} \hat{H}_{i, j}(\hat{x}) d \hat{x}=\int_{Q} \hat{H}_{i, j}(\hat{x}) d \hat{x}+R(\delta),
$$

where $R(\delta) \rightarrow 0$ as $\delta \rightarrow 0$.

(d) The function $\hat{H}_{i, j}(\hat{x})$ is analytic in $Q$.

Proof. The first assertion follows from (3.2) since, for fixed $\hat{x} \in Q$, the integrand is bounded and the finite part integral coincides with the usual Riemann integral. The second assertion follows from Proposition 1 and Fubini's theorem.

Expansion (5.7) follows from statement (b) as in [8, Chap. 6.1.3].

The analyticity of $\hat{H}_{i, j}$ is a direct consequence of (5.5).

This result will later be the base for the construction of the cubature method.

Case II: In the following, we will investigate the singular behaviour of $H_{i, j}$ in the case that $\overline{K_{i}}$ and $\overline{K_{j}}$ share exactly one edge. Without loss of generality we assume that the charts $\eta_{i}, \eta_{j}$ mapping the reference element $Q$ onto $K_{i}, K_{j}$ satisfy

$$
\eta_{i}\left(\begin{array}{l}
t \\
0
\end{array}\right)=\eta_{j}\left(\begin{array}{l}
t \\
0
\end{array}\right) \quad \forall t \in[0,1] .
$$

Hence, the difference

$$
z=\eta_{j}(\hat{y})-\eta_{i}(\hat{x})
$$

is zero if and only if the three-dimensional relative coordinates

$$
\hat{z}:=\left(\hat{y}_{1}-\hat{x}_{1}, \hat{y}_{2}, \hat{x}_{2}\right)^{T}
$$

equal zero. The difference $z$ then can be rewritten as

$$
z=\eta_{j}\left(\begin{array}{c}
\hat{x}_{1}+\hat{z}_{1} \\
\hat{z}_{2}
\end{array}\right)-\eta_{i}\left(\begin{array}{c}
\hat{x}_{1} \\
\hat{z}_{3}
\end{array}\right) \text {. }
$$

Using the abbreviations $r=\|\hat{z}\|$ and $\xi=\hat{z} / r$ and expanding $z$ about $r=0$ yields the representation

$$
z=\eta_{j}\left(\begin{array}{c}
\hat{x}_{1}+\hat{z}_{1} \\
\hat{z}_{2}
\end{array}\right)-\eta_{i}\left(\begin{array}{c}
\hat{x}_{1} \\
\hat{z}_{3}
\end{array}\right)=r \sum_{m=0}^{\infty} r^{m} \lambda_{m}\left(\hat{x}_{1}, \xi\right)
$$

with

$$
\lambda_{m}\left(\hat{x}_{1}, \xi\right)=\frac{\left\langle\xi_{12}, \nabla\right\rangle^{m+1} \eta_{j}\left(\begin{array}{c}
\hat{x}_{1} \\
0
\end{array}\right)-\left(\xi_{3} \partial_{2}\right)^{m+1} \eta_{i}\left(\begin{array}{c}
\hat{x}_{1} \\
0
\end{array}\right)}{(m+1) !} .
$$


Similarly as in Case I, the following expansion is derived

$$
k_{i, j}(\hat{x}, \hat{y})=r^{-s} b\left(\hat{x}_{1}, r, \xi\right),
$$

where $b\left(\hat{x}_{1}, r, \xi\right)$ is analytic with respect to

1. $\hat{x}_{1}$ in a neighbourhood of $(0,1)$,

2. $r$ in a neighbourhood of $\left\{r \in \mathbb{R} \mid \exists \hat{x}, \hat{y} \in Q: r^{2}=\hat{x}_{2}^{2}+\hat{y}_{2}^{2}+\left(\hat{y}_{1}-\hat{x}_{1}\right)^{2}\right\}$,

3. $\xi$ in a neighbourhood of $S_{2}$.

In contrast to the result of Proposition 1 the function $\hat{H}_{i, j}(\hat{x})$ contains nonintegrable singularities for $\hat{x}_{2} \rightarrow 0$. In this light, we will investigate the integrals

$$
\int_{Q^{\delta}} \hat{H}_{i, j}(\hat{x}) d \hat{x}
$$

as $\delta \rightarrow 0$ (cf. (4.4)). In Lemma 3 we will prove that $Q^{\delta}$ can be replaced by the simpler domain $\tilde{Q}^{\delta}$ defined by

$$
\tilde{Q}^{\delta}:=\left\{\hat{x} \in Q \mid \hat{x}_{2}>\delta\right\} .
$$

Lemma 3. The difference

$$
R(\delta):=\int_{Q^{\delta}} \hat{H}_{i, j}(\hat{x}) d \hat{x}-\int_{\tilde{Q}^{\delta}} \hat{H}_{i, j}(\hat{x}) d \hat{x}
$$

converges to zero as $\delta \rightarrow 0$.

Proof. Let $Q^{I}$ denote the triangle with vertices $(0,0)^{T},(1,0)^{T}$, and $(1 / 4,1 / 4)^{T}$. The complement is denoted by $Q^{I I}:=Q \backslash \overline{Q^{I}}$. It suffices to prove that $\hat{H}_{i, j}$ is weakly singular on $Q^{I I}$. The assertion then follows from [8, Chap. 6.1.3]. From (5.10), it follows

$$
\left|\hat{H}_{i, j}(\hat{x})\right| \leq \int_{Q}\left|k_{i, j}(\hat{x}, \hat{y})\right| d \hat{y} \leq \int_{0}^{1} \int_{0}^{1} \frac{c}{\left(\hat{x}_{2}^{2}+\left(\hat{y}_{1}-\hat{x}_{1}\right)^{2}+\hat{y}_{2}^{2}\right)^{3 / 2}} d \hat{y} .
$$

Introducing polar coordinates about $\left(\hat{x}_{1}, 0\right)^{T}$ results in

$$
\left|\hat{H}_{i, j}(\hat{x})\right| \leq \int_{0}^{\pi} \int_{0}^{R\left(\alpha, \hat{x}_{1}\right)} \frac{c r}{\left(\hat{x}_{2}^{2}+r^{2}\right)^{3 / 2}} d r d \alpha,
$$

where $R\left(\alpha, \hat{x}_{1}\right)$ denotes the upper limit of the $r$-integration. Performing the $r$ integration analytically yields

$$
\left|\hat{H}_{i, j}(\hat{x})\right| \leq c \int_{0}^{\pi} \frac{1}{\hat{x}_{2}}-\frac{1}{\sqrt{\hat{x}_{2}^{2}+R\left(\alpha, \hat{x}_{1}\right)^{2}}} d \alpha \leq \frac{\pi c}{\hat{x}_{2}} .
$$

The weak singularity of $\hat{H}_{i, j}$ on $Q^{I I}$ follows from

$$
\int_{Q^{I I}}\left|\hat{H}_{i, j}(\hat{x})\right| d \hat{x} \leq \int_{0}^{1} \int_{\hat{x}_{1}}^{1} \frac{\pi c}{\hat{x}_{2}} d \hat{x}+\int_{0}^{1} \int_{\left(1-\hat{x}_{1}\right) / 3}^{1} \frac{\pi c}{\hat{x}_{2}} d \hat{x}=\pi c(2+\ln 3) .
$$

In view of (4.4), we have to show that

$$
I(\delta):=\int_{\tilde{Q}^{\delta}} \int_{Q} k_{i, j}(\hat{x}, \hat{y}) d \hat{y} d \hat{x}
$$


admits an expansion of the form

$$
I(\delta)=I_{\log } \log \delta+I_{0}+I_{1}(\delta),
$$

where $I_{1}(\delta)$ converges to zero as $\delta \rightarrow 0$. Since the integrand in (5.12) is analytic, the transformation rule of variable applies and the domain of integration can be split into appropriate subdomains. We introduce relative coordinates by

$$
\left(\begin{array}{l}
\hat{x}_{1} \\
\hat{x}_{2} \\
\hat{y}_{1} \\
\hat{y}_{2}
\end{array}\right):=M \hat{z}:=\left(\begin{array}{cccc}
1 & 0 & 0 & 0 \\
0 & 1 & 0 & 0 \\
1 & 0 & 1 & 0 \\
0 & 0 & 0 & 1
\end{array}\right)\left(\begin{array}{c}
\hat{z}_{1} \\
\hat{z}_{2} \\
\hat{z}_{3} \\
\hat{z}_{4}
\end{array}\right) .
$$

As an abbreviation we write $\hat{k}(\hat{z})=k_{i, j}\left((M \hat{z})_{12},(M \hat{z})_{34}\right)$. The domain of integration is given by

$$
D=\left\{\begin{array}{c}
\delta \leq \hat{z}_{2} \leq 1 \\
0 \leq \hat{z}_{1} \leq 1-\hat{z}_{2} \\
-\hat{z}_{1} \leq \hat{z}_{3} \leq 1-\hat{z}_{1} \\
0 \leq \hat{z}_{4} \leq 1-\hat{z}_{3}-\hat{z}_{1}
\end{array}\right\}
$$

The integrand $\hat{k}$ is singular only if $\left(\hat{z}_{1}, \hat{z}_{2}, \hat{z}_{3}\right)^{T}=0$ (cf. (5.9)). Since $\hat{k}$ is smooth with respect to $\hat{z}_{1}$, we interchange the ordering of integration such that $\hat{z}_{1}$ becomes the innermost integration (cf. [23] and 9]). In order to characterize $D$ by a system of inequalities, where $\hat{z}_{1}$ stands at the last position, one has to split $D$ into five subdomains:

$$
\begin{aligned}
D & =\bigcup_{m=1}^{5} D_{m}, \\
I(\delta) & =\sum_{m=1}^{5} \int_{D_{m}} \hat{k}(\hat{z}) d \hat{z} .
\end{aligned}
$$

These subdomains are given explicitly below:

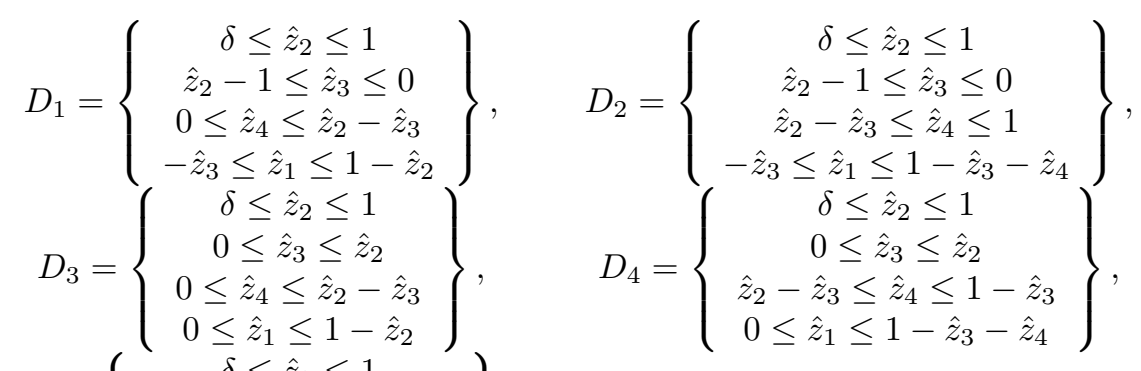

$$
\begin{aligned}
& D_{5}=\left\{\begin{array}{c}
\delta \leq \hat{z}_{2} \leq 1 \\
\hat{z}_{2} \leq \hat{z}_{3} \leq 1 \\
0 \leq \hat{z}_{4} \leq 1-\hat{z}_{3} \\
0 \leq \hat{z}_{1} \leq 1-\hat{z}_{3}-\hat{z}_{4}
\end{array}\right\} \text {. }
\end{aligned}
$$

By applying suitable four-dimensional rotations these subdomains can be mapped onto four-dimensional polyhedrons having the property that the origin is a corner 
point. We will need the following reference elements:

$$
\hat{D}_{1}=\left\{\begin{array}{c}
\delta \leq v_{1} \leq 1 \\
\delta \leq v_{2} \leq v_{1} \\
\delta \leq v_{3} \leq v_{2} \\
v_{1} \leq v_{4} \leq 1 \\
\delta \leq v_{1} \leq 1 \\
0 \leq v_{2} \leq v_{1} \\
0 \leq v_{3} \leq v_{2} \\
v_{1} \leq v_{4} \leq 1
\end{array}\right\}, \quad \hat{D}_{2}=\left\{\begin{array}{c}
\delta \leq v_{1} \leq 1 \\
\delta \leq v_{2} \leq v_{1} \\
0 \leq v_{3} \leq v_{2} \\
v_{1} \leq v_{4} \leq 1 \\
\delta \leq v_{1} \leq 1 \\
\delta \leq v_{2} \leq v_{1} \\
0 \leq v_{3} \leq v_{1} \\
v_{1} \leq v_{4} \leq 1
\end{array}\right\} .
$$

The integral over $D$ can be rewritten as

$$
I(\delta)=\int_{D} \hat{k}(\hat{z}) d \hat{z}=\sum_{m=1}^{4} \int_{\hat{D}_{m}} \hat{k}_{m}(v) d v .
$$

The functions $\hat{k}_{m}$ are defined by

$$
\hat{k}_{m}(v):= \begin{cases}\hat{k}\left(M_{1} v\right)+\hat{k}\left(M_{2} v\right), & m=1 \\ \hat{k}\left(M_{m+1} v\right), & m=2,3,4\end{cases}
$$

with

$$
\begin{array}{ll}
M_{1}=\left[\begin{array}{cccc}
-1 & 1 & -1 & 1 \\
0 & 0 & 1 & 0 \\
0 & -1 & 1 & 0 \\
1 & 0 & 0 & 0
\end{array}\right], & M_{2}=\left[\begin{array}{cccc}
-1 & 0 & 0 & 1 \\
0 & 0 & 1 & 0 \\
0 & 1 & 0 & 0 \\
1 & -1 & 0 & 0
\end{array}\right], \\
M_{3}=\left[\begin{array}{cccc}
-1 & 0 & 0 & 1 \\
0 & 1 & 0 & 0 \\
0 & 0 & 1 & 0 \\
1 & 0 & -1 & 0
\end{array}\right], & M_{4}=\left[\begin{array}{cccc}
-1 & 0 & 0 & 1 \\
1 & 0 & 0 & 0 \\
0 & 0 & 1 & 0 \\
0 & 1 & -1 & 0
\end{array}\right], \\
M_{5} & =\left[\begin{array}{cccc}
0 & -1 & 0 & 1 \\
0 & 1 & 0 & 0 \\
-1 & 1 & 0 & 0 \\
0 & 0 & 1 & 0
\end{array}\right] .
\end{array}
$$

The linearity of the transformations $M_{m}$ implies that the kernel function $\hat{k}_{m}$ is singular if and only if $\left(v_{1}, v_{2}, v_{3}\right)^{T}=0$ (cf. (5.9)). Furthermore, the smoothness behaviour of $\hat{k}_{m}$ can be derived from the smoothness behaviour of the function $k_{i, j}$ of (5.10). In particular, $\hat{k}_{m}$ is analytic in the variable $v_{4}$ (which corresponds to the behaviour of $k_{i, j}(\hat{x}, \hat{y})$ with respect to $\left.\hat{x}_{1}\right)$. Hence, the innermost integration (with respect to $\left.v_{4}\right)$ in (5.15) defines a function $\check{\kappa}_{m}\left(v_{1}, v_{2}, v_{3}\right)$ which has the same smoothness behaviour as $k_{i, j}$ as a function of $\hat{z}$ (see (5.9)). Let $\hat{D}_{m}^{-}$denote the domain $\hat{D}_{m}$ reduced by the last variable,

$$
\hat{D}_{m}^{-}:=\left\{w \in \mathbb{R}^{3} \mid \exists v \in \hat{D}_{m}, \forall i=1,2,3: w_{i}=v_{i}\right\},
$$

resulting in

$$
I(\delta)=\sum_{m=1}^{4} \int_{\hat{D}_{m}} \hat{k}_{m}(v) d v=\sum_{m=1}^{4} \int_{\hat{D}_{m}^{-}} \check{\kappa}_{m}(w) d w .
$$


In order to describe the domains $\hat{D}_{m}^{-}$in spherical coordinates $(r, \alpha, \beta)$, i.e.,

$$
w=r \psi(\alpha, \beta)=r\left(\begin{array}{c}
\cos \alpha \cos \beta \\
\sin \alpha \cos \beta \\
\sin \beta
\end{array}\right),
$$

we will employ the three-dimensional parameter domains $P_{\theta, m}(\delta), 1 \leq \theta \leq 3$, $1 \leq m \leq 2$. Let

$$
\begin{array}{ll}
a_{1}=0, & a_{2}=a_{3}=\arctan \delta \\
b_{1}(\alpha)=b_{2}(\alpha)=0, & b_{3}(\alpha):=\arctan (\delta \cos \alpha) \\
B_{1}(\alpha):=\arctan \cos \alpha, & B_{2}(\alpha):=\arctan \sin \alpha
\end{array}
$$

Then

$$
P_{\theta, m}=\left\{\begin{array}{c}
\alpha_{\theta} \leq \alpha \leq \frac{\pi}{4} \\
b_{\theta}(\alpha) \leq \beta \leq B_{m}(\alpha) \\
\frac{\delta}{\psi_{\theta}(\alpha, \beta)} \leq r \leq \frac{1}{\cos \alpha \cos \beta}
\end{array}\right\}
$$

We do not indicate explicitly the dependence of $P_{\theta, m}$ on $\delta$, while, for $\delta=0$, we write $P_{\theta, m}^{0}$ instead. Then, the integral (5.15) can be written in the following form:

$$
I(\delta)=\int_{D} \hat{k}(v) d v=\sum_{m=1}^{4} \int_{\check{D}_{m}^{-}} \check{\kappa}_{m}(r \psi) r^{2} \cos \beta d r d \beta d \alpha
$$

with

$$
\begin{array}{ll}
\check{D}_{1}^{-}=P_{3,2}, & \check{D}_{2}^{-}=P_{2,2}, \\
\check{D}_{3}^{-}=P_{1,2}, & \check{D}_{4}^{-}=P_{2,1} .
\end{array}
$$

Since the transformation of the spherical coordinates appearing in (5.18) onto the coordinates (5.10) is analytic, the smoothness and singular behaviour of the function $\hat{\kappa}_{m}(r \psi)$ carries over from the behaviour of $k_{i, j}$ (see (5.10) and the remarks thereafter). This means that the integrand in (5.18) can be represented by

$$
r^{2} \check{\kappa}_{m}(r \psi) \cos \beta=\frac{\check{\kappa}_{m}^{0}(\psi)}{r}+\check{\kappa}_{m}^{1}(r, \psi),
$$

where

$$
\begin{aligned}
\check{\kappa}_{m}^{0}(\psi) & =\lim _{r \rightarrow 0}\left(r^{3} \breve{\kappa}_{m}(r \psi) \cos \beta\right), \\
\check{\kappa}_{m}^{1}(r, \psi) & =r^{2} \check{\kappa}_{m}(r \psi) \cos \beta-\check{\kappa}_{m}^{0}(\psi) / r
\end{aligned}
$$

are analytic with respect to $r, \alpha, \beta$. The domain $\check{D}_{m}^{-}$depends on $\delta$. Since $\check{\kappa}_{m}^{1}$ is analytic, the coefficient $I_{\log }$ in (5.13) depends only on the first sum below while the second stays bounded as $\delta \rightarrow 0$

$$
I(\delta)=\sum_{m=1}^{4} \int_{\check{D}_{m}^{-}} \frac{\check{\kappa}_{m}^{0}(\psi)}{r} d r d \beta d \alpha+\sum_{m=1}^{4} \int_{\check{D}_{m}^{-}} \check{\kappa}_{m}^{1}(r, \psi) d r d \beta d \alpha .
$$

The $r$-integration can be carried out analytically for the first integral above. We obtain

$$
\begin{aligned}
\int_{\check{D}_{m}^{-}} \frac{\check{\kappa}_{m}^{0}(\psi)}{r} d r d \beta d \alpha= & -\log \delta \int_{a_{\theta}}^{\pi / 4} \int_{b_{\theta}(\alpha)}^{B_{t}(\alpha)} \check{\kappa}_{m}^{0}(\psi) d \beta d \alpha \\
& +\int_{a_{\theta}}^{\pi / 4} \int_{b_{\theta}(\alpha)}^{B_{t}(\alpha)} \check{\kappa}_{m}^{0}(\psi) \log \frac{\psi_{\theta}(\alpha, \beta)}{\cos \alpha \cos \beta} d \beta d \alpha
\end{aligned}
$$


where $a_{\theta}, b_{\theta}, B_{t}$ are as in (5.17) and $(\theta, t)$ is determined by

$$
\check{D}_{m}^{-}=P_{\theta, t}
$$

(see (5.19) ). The first integral defines the coefficient $I_{\log , m}$ while the second integrand is weakly singular. From [8, Chap. 6.1.3], it follows that

$$
\begin{aligned}
& \int_{\check{D}_{m}^{-}} \frac{\check{\kappa}_{m}^{0}(\psi)}{r} d r d \beta d \alpha \\
& \quad=I_{\log , m} \log \delta+\int_{0}^{\pi / 4} \int_{0}^{B_{t}(\alpha)} \check{\kappa}_{m}^{0}(\psi) \log \frac{\psi_{\theta}(\alpha, \beta)}{\cos \alpha \cos \beta} d \beta d \alpha+I_{1, m}(\delta)
\end{aligned}
$$

holds, where $I_{1, m}(\delta) \rightarrow 0$ as $\delta \rightarrow 0$. The considerations above are summarized in the next lemma.

Lemma 4. Let $K_{i}, K_{j} \in \tau$ share exactly one edge. Then

$$
\int_{Q^{\delta}} \hat{H}_{i, j}(\hat{x}) d \hat{x}=I_{\log } \log \delta+I_{0}+I_{1}(\delta)
$$

holds, where $I_{1}(\delta) \rightarrow 0$ as $\delta \rightarrow 0$ and

$$
\begin{aligned}
& I_{\log }=\sum_{m=1}^{4} I_{\log , m}, \\
& I_{0}=\sum_{m=1}^{4} \int_{0}^{\pi / 4} \int_{0}^{B_{t}(\alpha)} \check{\kappa}_{m}^{0}(\psi) \log \frac{\psi_{\theta}(\alpha, \beta)}{\cos \alpha \cos \beta} d \beta d \alpha+\int_{\check{D}_{m}^{0}} \check{\kappa}_{m}^{1}(r \psi) d r d \beta d \alpha .
\end{aligned}
$$

The function $B_{t}$ and indices $(\theta, t)$ are defined as in (5.22), and $\check{D}_{m}^{0}$ is obtained by setting $\delta=0$ in the definition of $P_{\theta, t}$.

Proof. The proof follows from the representation (5.23).

Cubature rules for computing the integrals appearing in the definition of $I_{0}$ will be presented in Section 6.

Case III: It remains to discuss the case of coinciding panels $K_{i}=K_{j}$. The function $\hat{H}_{i, j}$ is defined as a finite-part integral (cf. (3.4))

$$
\hat{H}_{i, i}(\hat{x}):=p . f \cdot \int_{Q} k_{i, i}(\hat{x}, \hat{y}) d \hat{y} .
$$

The integrand is singular only if $y=x$. The difference

$$
z=\eta_{i}(\hat{y})-\eta_{i}(\hat{x})
$$

is zero only if $\hat{y}=\hat{x}$. In this light, we introduce the following two-dimensional relative coordinates (cf. [23], [9])

$$
\hat{z}=\hat{y}-\hat{x}
$$

resulting in

$$
z=\eta_{i}(\hat{x}+\hat{z})-\eta_{i}(\hat{x})
$$


Using the abbreviation $r=\|\hat{z}\|$ and $\xi=\hat{z} / r$ and expanding $z$ about $r=0$ yields the representation

$$
z=\eta_{i}(\hat{x}+r \xi)-\eta_{i}(\hat{x})=r \sum_{m=0}^{\infty} r^{m} \chi_{m}(\hat{x}, \xi),
$$

where

$$
\chi_{m}(\hat{x}, \xi)=\frac{\langle\xi, \nabla\rangle^{m+1} \eta_{i}(\hat{x})}{(m+1) !} .
$$

Similarly as in Case I, the following expansion is derived:

$$
k_{i, i}(\hat{x}, \hat{y})=r^{-s} c(\hat{x}, r, \xi),
$$

where $c(\hat{x}, r, \xi)$ is analytic with respect to

1. $\hat{x}$ in a neighbourhood of $Q$,

2. $r$ in a neighbourhood of $\{r \in \mathbb{R} \mid \hat{x}, \hat{y} \in Q: r=\|\hat{y}-\hat{x}\|\}$,

3. $\xi$ in a neighbourhood of $S_{1}$.

Due to the analyticity of $c$ we can define the following functions:

$$
\begin{aligned}
c_{0}(\hat{x}, \xi) & =\lim _{r \rightarrow 0} c(\hat{x}, r, \xi) \\
c_{1}(\hat{x}, \xi) & =\lim _{r \rightarrow 0} \partial_{r} c(\hat{x}, r, \xi) \\
k_{r e g}(\hat{x}, \hat{x}+r \xi) & =\frac{c(\hat{x}, r, \xi)-c_{0}(\hat{x}, \xi)-r c_{1}(\hat{x}, \xi)}{r^{s}} .
\end{aligned}
$$

In view of the analyticity of the coefficients $c, c_{0}, c_{1}$, and of the kernel function, $k_{r e g}(\hat{x}, \hat{x}+r \xi)$ can be written as

$$
k_{r e g}(\hat{x}, \hat{x}+r \xi)=\frac{\tilde{c}(\hat{x}, r, \xi)}{r}
$$

(recall $s \leq 3$ ), where $\tilde{c}$ has the same analyticity behaviour as the function $c$ from (5.24). Similarly as worked out in Proposition 1 and Lemma 2, one proves that

$$
\hat{H}_{i, i}^{r e g}(\hat{x}):=\int_{Q} k_{r e g}(\hat{x}, \hat{y}) d \hat{y}
$$

exists as an improper integral and is weakly singular with respect to $\hat{x}$. Altogether, the representation

$$
\begin{aligned}
\hat{H}_{i, i}(\hat{x}) & =p . f \cdot \int_{\mathcal{P}_{i}} \frac{c_{0}(\hat{x}, \psi(\alpha))}{r^{2}} d r d \alpha+p . f \cdot \int_{\mathcal{P}_{i}} \frac{c_{1}(\hat{x}, \psi(\alpha))}{r} d r d \alpha+\hat{H}_{i, i}^{r e g}(\hat{x}) \\
& =\hat{H}_{i, i}^{0}(\hat{x})+\hat{H}_{i, i}^{1}(\hat{x})+\hat{H}_{i, i}^{r e g}(\hat{x})
\end{aligned}
$$

is proved, where

$$
\psi(\alpha):=\left(\begin{array}{c}
\cos \alpha \\
\sin \alpha
\end{array}\right)
$$

and $\mathcal{P}_{i}$ denotes the domain $(Q-\hat{x})$ in polar coordinates

$$
\mathcal{P}_{i}=\left\{\begin{array}{c}
0 \leq \alpha \leq 2 \pi \\
\varepsilon \leq r \leq R(\alpha, \hat{x})
\end{array}\right\} .
$$


The function $R(\alpha, \hat{x})$ is defined as follows. Let $\left\{P_{m}\right\}_{m=1}^{3}$ denote the vertices of $Q$ (counterclockwise ordering and $P_{1}=(0,0)^{T}$ ), and $e_{m}=P_{m+1}-P_{m}$ the edges of $Q$. The distance of $\hat{x} \in Q$ from the $m$ th edge is given by

$$
d_{m}(\hat{x}):=\inf _{t \in(0,1)}\left\|P_{m}+t e_{m}-\hat{x}\right\|,
$$

and the auxiliary functions $a_{m}(\hat{x}), p_{m}(\alpha)$ by the following table.

\begin{tabular}{|l|l|l|}
\hline$m$ & $a_{m}(\hat{x})=$ & $p_{m}(\alpha)=$ \\
\hline 0 & $-\pi+\arctan \frac{\hat{x}_{2}}{\hat{x}_{1}}$ & \\
\hline 1 & $-\arctan \frac{\hat{x}_{2}}{1-\hat{x}_{1}}$ & $-\sin \alpha$ \\
\hline 2 & $\pi-\arctan \frac{1-\hat{x}_{2}}{\hat{x}_{1}}$ & $\frac{\cos \alpha+\sin \alpha}{\sqrt{2}}$ \\
\hline 3 & $\pi+\arctan \frac{\hat{x}_{2}}{\hat{x}_{1}}$ & $-\cos \alpha$ \\
\hline
\end{tabular}

Then, $R(\alpha, \hat{x})$ is given by

$$
R(\alpha, \hat{x})=\frac{d_{m}(\hat{x})}{p_{m}(\alpha)} \quad \forall \alpha \in\left(a_{m-1}(\hat{x}), a_{m}(\hat{x})\right) .
$$

The $r$-integration in the definition of $\hat{H}_{i, i}^{0}$ and $\hat{H}_{i, i}^{1}$ can be performed analytically and the finite-part process as well:

$$
\begin{aligned}
\hat{H}_{i, i}^{0}(\hat{x}) & =p \cdot f \cdot \int_{0}^{2 \pi} \int_{\varepsilon}^{R(\hat{x}, \alpha)} \frac{c_{0}(\hat{x}, \psi(\alpha))}{r^{2}} d r d \alpha=-\int_{0}^{2 \pi} \frac{c_{0}(\hat{x}, \psi(\alpha))}{R(\hat{x}, \alpha)} d \alpha \\
& =-\sum_{m=1}^{3} \frac{1}{d_{m}(\hat{x})} \int_{a_{m-1}(\hat{x})}^{a_{m}(\hat{x})} p_{m}(\alpha) c_{0}(\hat{x}, \psi(\alpha)) d \alpha=: \sum_{m=1}^{3} \frac{C_{m}(\hat{x})}{d_{m}(\hat{x})} \\
\hat{H}_{i, i}^{1}(\hat{x}) & =\sum_{m=1}^{3} \int_{a_{m-1}(\hat{x})}^{a_{m}(\hat{x})} c_{1}(\hat{x}, \psi(\alpha)) \log \frac{d_{m}(\hat{x})}{p_{m}(\alpha)} d \alpha .
\end{aligned}
$$

The properties of the functions $\hat{H}_{i, i}^{0}$ and $\hat{H}_{i, i}^{1}$ are collected in the following lemma.

Lemma 5. The integrands in (5.30) and (5.31) are weakly singular (with respect to $\alpha$ ).

The function $\hat{H}_{i, i}^{1}$ is weakly singular while the function $\hat{H}_{i, i}^{0}$ contains nonintegrable singularities as $x \rightarrow \partial Q$. The function $C_{m}$ in (5.30) is analytic. More precisely, the function $\frac{C_{m}}{d_{m}}$ is strongly singular only if $\hat{x} \rightarrow \overline{P_{m} P_{m+1}}$ and analytic in $Q$.

Proof. These statements follow directly from (5.30) and the analyticity of $p_{m}$ and $c_{0}$.

Next, we have to prove that

$$
\int_{Q^{\delta}} \hat{H}_{i, i}(\hat{x}) d \hat{x}=I_{\log } \log \delta+I_{0}+I_{1}(\delta)
$$

holds with $I_{1}(\delta) \rightarrow 0$ as $\delta \rightarrow 0$. Similarly as in the case of a common edge (cf. Lemma (3), this problem can be simplified. Let $\tilde{Q}^{\delta}$ be defined as in (5.11). For 
$m=1,2,3$, the mapping $W_{m}: Q \rightarrow Q$ and the numbers $g_{m}$ are given by (notation: $P_{4}:=P_{1}$ and $\left.P_{5}:=P_{2}\right)$

$$
\begin{gathered}
W_{m}(\hat{x})=P_{m}+\left(P_{m+1}-P_{m}\right) \hat{x}_{1}+\left(P_{m+2}-P_{m}\right) \hat{x}_{2}, \\
g_{m}:= \begin{cases}\sqrt{2} & \text { if } m=2, \\
1 & \text { otherwise. }\end{cases}
\end{gathered}
$$

Lemma 6. The difference

$R(\delta):=\int_{Q^{\delta}} \hat{H}_{i, i}(\hat{x}) d \hat{x}-\left\{\int_{Q} \hat{H}_{i, i}^{r e g}(\hat{x})+\hat{H}_{i, i}^{1}(\hat{x}) d \hat{x}+\sum_{m=1}^{3} g_{m} \int_{\tilde{Q}^{\delta}} \frac{C_{m}\left(W_{m} \hat{x}\right)}{\hat{x}_{2}} d \hat{x}\right\}$ converges to zero as $\delta \rightarrow 0$.

Proof. We have to show that

$$
\tilde{R}(\delta):=g_{m} \int_{\tilde{Q}^{\delta}} \frac{C_{m}\left(W_{m} \hat{x}\right)}{\hat{x}_{2}} d \hat{x}-\int_{Q^{\delta}} \frac{C_{m}(\hat{x})}{d_{m}(\hat{x})} d \hat{x}
$$

converges to zero as $\delta \rightarrow 0$. We observe that $W_{m}$ maps $Q^{\delta}$ onto $Q^{\delta}$. Since $d_{m}\left(W_{m} \hat{x}\right)=\hat{x}_{2} / g_{m}$, it suffices to proof that

$$
\left\{\int_{\tilde{Q}^{\delta}} \frac{C_{m}\left(W_{m} \hat{x}\right)}{\hat{x}_{2}} d \hat{x}-\int_{Q^{\delta}} \frac{C_{m}\left(W_{m} \hat{x}\right)}{\hat{x}_{2}} d \hat{x}\right\} \stackrel{\delta \rightarrow 0}{\rightarrow} 0 .
$$

The proof of (5.34) follows by the same arguments as the proof of Lemma 3 ,

Now we can prove that (5.32) holds.

Lemma 7. The integral $\int_{Q^{\delta}} \hat{H}_{i, i}(\hat{x}) d \hat{x}$ can be written as

$$
\int_{Q^{\delta}} \hat{H}_{i, i}(\hat{x}) d \hat{x}=I_{\log } \log \delta+I_{0}+I_{1}(\delta)
$$

where $I_{1}(\delta) \rightarrow 0$ as $\delta \rightarrow 0$.

Proof. Due to the analyticity of $C_{m}$ the functions

$$
\begin{aligned}
C_{m}^{0}\left(\hat{x}_{1}\right): & =\lim _{\hat{x}_{2} \rightarrow 0} g_{m} C_{m}\left(W_{m} \hat{x}\right), \\
C_{m}^{1}(\hat{x}): & =\frac{g_{m} C_{m}\left(W_{m} \hat{x}\right)-C_{m}^{0}\left(\hat{x}_{1}\right)}{\hat{x}_{2}}
\end{aligned}
$$

are analytic too. Therefore

$$
\begin{aligned}
g_{m} \int_{\tilde{Q}_{i}^{\delta}} \frac{C_{m}\left(W_{m} \hat{x}\right)}{\hat{x}_{2}} d \hat{x}= & -\log \delta \int_{0}^{1} C_{m}^{0}\left(\hat{x}_{1}\right) d \hat{x}_{1}+\int_{0}^{1} C_{m}^{0}\left(\hat{x}_{1}\right) \log \left(1-\hat{x}_{1}\right) d \hat{x}_{1} \\
& +\int_{Q} C_{m}^{1}(\hat{x}) d \hat{x}+R(\delta)
\end{aligned}
$$

where $R(\delta) \rightarrow 0$ as $\delta \rightarrow 0$.

As a side result we obtain a statement on the analyticity of $\hat{H}_{i, j}$.

Lemma 8. The function $\hat{H}_{i, j}(\hat{x})$ is analytic in $Q$ and is possibly singular as $\hat{x} \rightarrow$ $\partial Q$. 
Proof. Let $\bar{B} \subset Q$. For $K_{i} \neq K_{j}$, the function $k_{i, j}: B \times Q \rightarrow \mathbb{C}$ is analytic since $\operatorname{dist}\left(\eta_{i}(B), K_{j}\right)>0$. Hence, $H_{i, j}(\hat{x})=\int_{Q} k_{i, j}(\hat{x}, \hat{y}) d \hat{y}$ is analytic in $B$. by

Now, let $K_{i}=K_{j}$. We employ the splitting (5.29). The term $\hat{H}_{i, i}^{\text {reg }}$ was defined

$$
\hat{H}_{i, i}^{r e g}(\hat{x}):=\int_{0}^{2 \pi} \int_{0}^{R(\alpha, x)} \tilde{c}\left(\hat{x}, r,\left(\begin{array}{c}
\cos \alpha \\
\sin \alpha
\end{array}\right)\right) d r d \alpha
$$

with $\tilde{c}$ as in (5.28). The integrand is analytic in all variables and the upper bound $R(\alpha, \hat{x})$ for the $r$-integration is also analytic in $Q$. Hence, the same holds for $\hat{H}_{i, i}^{r e g}$.

The term $\hat{H}_{i, i}^{0}$ can be written in the form $\sum_{m=1}^{\nu} \frac{C_{m}(\hat{x})}{d_{m}(\hat{x})}$ with analytic $C_{m}$ and $d_{m}$ denoting the distance to the $m$ th edge. Hence, $\hat{H}_{i, i}^{0}$ has also the asserted analyticity properties.

Taylor expansion of the function $c_{0}(\hat{x}, \psi(\alpha))$ of (5.31) with respect to $\hat{x}$ and integrating the coefficients with respect to $\alpha$ implies that $\hat{H}_{i, i}^{1}$ has the asserted analyticity properties too.

The following remark concerns the extension of the analysis presented to the case that the kernel function contains stronger singularities: $s>3$. However, we emphasize that, in most applications in $\mathbb{R}^{3}, s \leq 3$ holds and the modifications below are irrelevant.

Remark 4. Let the kernel function be of the form (3.2) with $s>3$. If $\overline{K_{i}}, \overline{K_{j}}$ share exactly one common point, the kernel function in (5.5) is no longer integrable. One has to subtract a Taylor expansion of $a_{4}(r, \xi)$ about $r=0$ (of order $s-3$ ), i.e., introduce a further finite part process similarly as in the case of a common edge and $s=3$.

In the case of a common edge or for identical panels, the orders of the Taylor expansions appearing in (5.21), (5.27), and (5.36) have to be increased. Furthermore, the replacement of the domain $Q^{\delta}$ by the simplified domains $\tilde{Q}^{\delta}$ are no longer possible due to the arising stronger corner singularities. Instead one has to carry out the integration over $Q^{\delta}$ explicitly and introduce appropriate regularisations for both edge and corner singularities.

\section{Cubature techniques}

In this section, we will define families of cubature rules for the approximation of the local, regularised integrals

$$
p \cdot f \cdot \int_{Q} p \cdot f \cdot \int_{Q} k_{i, j}(\hat{x}, \hat{y}) d \hat{y} d \hat{x}
$$

appearing in the sum (4.5). We distinguish the following four cases:

1. $\overline{K_{i}} \cap \overline{K_{j}}=\emptyset$,

2. $\overline{K_{i}}, \overline{K_{j}}$ share exactly one point,

3. $\overline{K_{i}}, \overline{K_{j}}$ share exactly one edge,

4. $K_{i}=K_{j}$.

Case 1: In the first case, the integrand is analytic and both finite part integrals in (6.1) reduce to usual Riemann integrals. Thus, four-dimensional tensor versions of properly scaled Gauß-Legendre quadrature rules are converging exponentially towards the true integral. 
Case 2: The integrand in this case is weakly singular, and we can write

$$
\int_{Q \times Q} k_{i, j}(\hat{x}, \hat{y}) d \hat{y} d \hat{x}
$$

The domain of integration is given by

$$
\begin{aligned}
& 0 \leq \hat{x}_{1} \leq 1 \\
& 0 \leq \hat{x}_{2} \leq 1-\hat{x}_{1}, \\
& 0 \leq \hat{y}_{1} \leq 1 \\
& 0 \leq \hat{y}_{2} \leq 1-\hat{y}_{1} .
\end{aligned}
$$

As explained in [4], 23] the transformations

$$
\chi^{I}(\omega, \eta):=\left(\begin{array}{c}
\eta_{1} \omega \\
\omega\left(1-\eta_{1}\right) \\
\eta_{2} \eta_{3} \omega \\
\eta_{2} \omega\left(1-\eta_{3}\right)
\end{array}\right), \quad \chi^{I I}(\omega, \eta):=\left(\begin{array}{c}
\eta_{2} \eta_{3} \omega \\
\eta_{2} \omega\left(1-\eta_{3}\right) \\
\eta_{1} \omega \\
\omega\left(1-\eta_{1}\right)
\end{array}\right)
$$

map the four-dimensional unit cube onto two disjoint domains $D_{1}, D_{2}$ satisfying $Q \times Q=D_{1} \cup D_{2}$. The Jacobian of both mappings is given by $\omega^{3} \eta_{2}$. The integral in (6.2) becomes

$$
\int_{(0,1)^{4}}\left\{k_{i, j}\left(\chi_{12}^{I}, \chi_{13}^{I}\right)+k_{i, j}\left(\chi_{12}^{I I}, \chi_{13}^{I I}\right)\right\} \omega^{3} \eta_{2} d \omega d \eta .
$$

Since the transform of these coordinates onto the polar coordinates (5.2) is analytic, the integrand in (6.3) is analytic, too (cf. [8, Remark 9.4.2]). Thus, fourdimensional Gauß-Legendre formulae defines an exponentially convergent family of cubature rules for approximating (6.2). Quantitative estimates of the local cubature errors along with formulae for the cubature orders required for a consistent discretisation will be presented in a forthcoming paper.

We expect that the integral 6.2 can be treated with extrapolation techniques as well (cf. [16], [15], 17], 26]), although the corresponding error expansions are not worked out yet.

Case 3: Now, we consider the case where $\overline{K_{i}}$ and $\overline{K_{j}}$ share exactly one edge. The following representation was proved in Lemma 4 where the quantities $\lambda, B_{m}$, $\check{\kappa}_{m}^{0}, \check{\kappa}_{m}^{1}, \psi, \check{D}_{m}^{0}$ are also defined (for $(\theta, t)$, see (5.19) and (5.22) $)$ :

$$
\begin{aligned}
p . f . \int_{Q} p . f . \int_{Q} k_{i, j}(\hat{x}, \hat{y}) d \hat{y} d \hat{x}= & \sum_{m=1}^{4} \int_{0}^{\pi / 4} \int_{0}^{B_{t}(\alpha)} \check{\kappa}_{m}^{0}(\psi) \log \frac{\psi_{\theta}(\alpha, \beta)}{\psi_{1}(\alpha, \beta)} d \beta d \alpha \\
& +\int_{\check{D}_{m}^{0}} \check{\kappa}_{m}^{1}(r \psi) d r d \beta d \alpha .
\end{aligned}
$$

It was proved that the functions $\check{\kappa}_{m}^{0}$ and $\check{\kappa}_{m}^{1}$ are analytic. The domain of integration $\check{D}_{m}^{0}$ is given by

$$
\begin{aligned}
& 0 \leq \alpha \leq \frac{\pi}{4} \\
& 0 \leq \beta \leq B_{t}(\alpha) \\
& 0 \leq r \leq \frac{1}{\cos \alpha \cos \beta}
\end{aligned}
$$


The integration bounds depend analytically on the parameters. Hence, the second integral on the right-hand side above can be approximated by tensor versions of properly scaled Gauß-Legendre quadrature rules. This family of quadrature methods again is exponentially convergent with respect to the order.

For the first integral on the right-hand side of (6.4), we observe that

- for $\theta=1$, the integral vanishes;

- for $\theta=2$, the integral can be rewritten as $\int_{0}^{\pi / 4} \log \tan \alpha \int_{0}^{B_{t}(\alpha)} \check{\kappa}_{m}^{0}(\psi) d \beta d \alpha$;

- for $\theta=3$, the integral takes the form

$$
\int_{0}^{\pi / 4} \int_{0}^{B_{t}(\alpha)} \check{\kappa}_{m}^{0}(\psi) \log \frac{\tan \beta}{\cos \alpha} d \beta d \alpha
$$

For $\theta=2$, the $\alpha$-integration can be approximated by Gauß-like formula with logarithmic weight (substituting $\tan \alpha=s$ ) as explained, e.g., in 11, while the integrand is analytic with respect to $\beta$. Hence, integration with respect to $\beta$ can be approximated with Gauß-Legendre quadrature rules.

$\theta=3$ implies $m=1$ and $t=2(\mathrm{cf}$. (5.19) $)$. In this case, we substitute the variable $\beta$ by

$$
\beta(\alpha, \xi)=\arctan (\xi \sin \alpha)
$$

Let the auxiliary function $\rho$ and $\psi$ be defined by

$$
\rho(\alpha, \xi):=\frac{\sin \alpha}{1+\xi^{2} \sin ^{2} \alpha}, \quad \psi(\alpha, \xi):=\frac{(\cos \alpha, \sin \alpha, \xi \sin \alpha)^{T}}{\sqrt{1+\xi^{2} \sin ^{2} \alpha}}
$$

Then, 6.5) can be rewritten as

$$
\int_{0}^{1} \log \xi \int_{0}^{\pi / 4} \rho \check{\kappa}_{m}^{0}(\psi) d \alpha d \xi+\int_{0}^{\pi / 4}(\log \tan \alpha) \int_{0}^{1} \rho \check{\kappa}_{m}^{0}(\psi) d \xi d \alpha
$$

Hence, Gauß-Legendre rules can be used with respect to those variables where the integrand is analytic and to Gauß-like rules with logarithmic weight with respect to the remaining variables.

We conclude this case by discussing how the function $\check{\kappa}_{m}^{0}$ (defined in (5.20) $)$,

$$
\check{\kappa}_{m}^{0}(\psi):=\lim _{r \rightarrow 0}\left(r^{3} \check{\kappa}_{m}(r \psi) \cos \beta\right)
$$

can be evaluated in quadrature points, where $\check{\kappa}$ was defined by (5.18). Since the expression in the brackets in (6.7) is analytic in $r$, the function $\kappa_{m}^{0}$ can be approximated for sufficiently small $\varepsilon>0$ by

$$
\widetilde{\kappa}_{m}^{0}(\psi):=\varepsilon^{3} \check{\kappa}_{m}(\varepsilon \psi) \cos \beta,
$$

where the arising error is proportional to $\varepsilon$. 
Case 4: In the case of identical panels we have proved that

$$
\begin{aligned}
& \underset{\delta}{p . f .} \int_{Q} p . f . \int_{Q} k_{i, i}(\hat{x}, \hat{y}) d \hat{y} d \hat{x}=\int_{Q \times Q} k_{r e g}(\hat{x}, \hat{y}) d \hat{y} d \hat{x} \\
& +\sum_{m=1}^{3}\left\{\int_{Q} \int_{a_{m-1}}^{a_{m}} c_{1}(\hat{x}, \psi(\alpha)) \log \frac{d_{m}(\hat{x})}{p_{m}(\alpha)} d \alpha d \hat{x}\right. \\
& \left.+\int_{Q} C_{m}^{1}(\hat{x}) d \hat{x}+\int_{0}^{1} C_{m}^{0}\left(\hat{x}_{1}\right) \log \left(1-\hat{x}_{1}\right) d \hat{x}_{1}\right\} \\
& =: I_{\text {reg }}+\sum_{m=1}^{3}\left\{I_{m}^{(1)}+I_{m}^{(2)}+I_{m}^{(3)}\right\}
\end{aligned}
$$

holds, where all quantities are defined in Section 4, Case III. For the approximation of $I_{\text {reg }}$, we employ the transformations and cubature techniques as described in [4] which goes back to [23].

The functions $C_{m}^{1}$ are analytic on $Q$ and, hence, $I_{m}^{(2)}$ can also be approximated by tensor versions of properly scaled Gauß-Legendre rules. The last integral $I_{m}^{(3)}$ can be approximated efficiently using Gauß-like formulas with weight $\log \left(1-\hat{x}_{1}\right)$.

It remains to consider the approximation of the integral $I_{m}^{(1)}$. We employ the transformation $W_{m}$, which was used in Lemma 6, and observe that $d_{m}\left(W_{m} \hat{x}\right)=$ $\hat{x}_{2} / g_{m}$ (cf. (5.33) ) holds. This means that

$$
\sum_{m=1}^{3} I_{m}^{(1)}=\sum_{m=1}^{3} \int_{Q} \int_{a_{m-1}\left(W_{m} \hat{x}\right)}^{a_{m}\left(W_{m} \hat{x}\right)} c_{1}\left(W_{m} \hat{x}, \psi(\alpha)\right) \log \frac{\hat{x}_{2}}{g_{m} p_{m}(\alpha)} d \alpha d \hat{x}
$$

holds, where the functions $a_{m}$ are as in (5.31). In the next step, this integral will be transformed onto a standard domain such that the singular behaviour of the integrand is simplified. Let the constant $\gamma_{m}$ be defined by

$$
\gamma_{1}=-\frac{\pi}{2}, \quad \gamma_{2}=\frac{\pi}{4}, \quad \gamma_{3}=\pi .
$$

Substituting $\alpha \leftarrow \gamma_{m}+\arctan \frac{\hat{z}}{\hat{x}_{2}}$, we obtain

$$
I_{m}^{(1)}=\int_{Q}^{\rho_{m}^{1}(\hat{x})} \int_{\rho_{m}^{0}(\hat{x})}^{\check{c}_{m}}(\hat{x}, \hat{z}) \check{\omega}_{m}(\hat{x}, \hat{z}) d \hat{z} d \hat{x}
$$

where the quantities $\rho_{m}, \check{c}_{m}$, and $\check{\omega}_{m}$ are defined by

$$
\begin{array}{ll}
\rho_{1}^{0}=-\hat{x}_{1}, & \rho_{1}^{1}=1-\hat{x}_{1}, \\
\rho_{2}^{0}=-2 \hat{x}_{1}-\hat{x}_{2}, & \rho_{2}^{1}=2-2 \hat{x}_{1}-\hat{x}_{2}, \\
\rho_{3}^{0}=-\hat{x}_{1}-\hat{x}_{2}, & \rho_{3}^{1}=1-\hat{x}_{1}-\hat{x}_{2}
\end{array}
$$

and

$$
\begin{aligned}
\check{c}_{m}(\hat{x}, \hat{z}): & =c_{1}\left(W_{m} \hat{x}, \psi\left(\gamma_{m}+\arctan \frac{\hat{z}}{\hat{x}_{2}}\right)\right), \\
\check{\omega}_{m}(\hat{x}, \hat{z}): & =\frac{\hat{x}_{2}}{\hat{x}_{2}^{2}+\hat{z}^{2}} \log \frac{\sqrt{\hat{z}^{2}+\hat{x}_{2}^{2}}}{g_{m}}
\end{aligned}
$$


Since the integrand in (6.8) is smooth with respect to $\hat{x}_{1}$, we interchange the ordering of integration such that $\hat{x}_{1}$ is the innermost variable. Again the integration domain has to be split into subdomains. Then the reference domain

$$
D_{1}:=\left\{\begin{array}{c}
0 \leq v_{1} \leq 1 \\
0 \leq v_{2} \leq v_{1} \\
0 \leq v_{3} \leq 1-v_{1}
\end{array}\right\}
$$

is mapped onto these subdomains. Let transformations $\chi_{m \mu}$ be defined as follows.

- For $m=1$, by

$$
\begin{aligned}
\chi_{11}(v) & =\left[\begin{array}{ccc}
1 & -1 & 1 \\
0 & 1 & 0 \\
-1 & 1 & 0
\end{array}\right]\left(\begin{array}{l}
v_{1} \\
v_{2} \\
v_{3}
\end{array}\right), \chi_{12}(v)=\left[\begin{array}{lll}
0 & 0 & 1 \\
1 & 0 & 0 \\
0 & 1 & 0
\end{array}\right]\left(\begin{array}{l}
v_{1} \\
v_{2} \\
v_{3}
\end{array}\right), \\
\chi_{13}(v) & =\left[\begin{array}{lll}
0 & 0 & 1 \\
0 & 1 & 0 \\
1 & 0 & 0
\end{array}\right]\left(\begin{array}{l}
v_{1} \\
v_{2} \\
v_{3}
\end{array}\right) .
\end{aligned}
$$

- For $m=2$, by

$$
\begin{array}{ll}
\chi_{21}(v) & =\left[\begin{array}{ccc}
0 & 0 & 1 \\
1 & 0 & 0 \\
-1 & 2 & 0
\end{array}\right]\left(\begin{array}{l}
v_{1} \\
v_{2} \\
v_{3}
\end{array}\right), \quad \chi_{22}(v)=\left[\begin{array}{ccc}
0 & 0 & 1 \\
0 & 1 & 0 \\
2 & -1 & 0
\end{array}\right]\left(\begin{array}{l}
v_{1} \\
v_{2} \\
v_{3}
\end{array}\right), \\
\chi_{23}(v) & =\left[\begin{array}{ccc}
1 & -1 & 1 \\
0 & 1 & 0 \\
-2 & 1 & 0
\end{array}\right]\left(\begin{array}{l}
v_{1} \\
v_{2} \\
v_{3}
\end{array}\right) .
\end{array}
$$

- For $m=3$, by

$$
\begin{array}{ll}
\chi_{31}(v) & =\left[\begin{array}{ccc}
1 & 0 & 1 \\
0 & 1 & 0 \\
-1 & 0 & 0
\end{array}\right]\left(\begin{array}{l}
v_{1} \\
v_{2} \\
v_{3}
\end{array}\right), \chi_{32}(v)=\left[\begin{array}{ccc}
0 & 0 & 1 \\
1 & 0 & 0 \\
0 & -1 & 0
\end{array}\right]\left(\begin{array}{l}
v_{1} \\
v_{2} \\
v_{3}
\end{array}\right), \\
\chi_{33}(v) & =\left[\begin{array}{ccc}
0 & 0 & 1 \\
0 & 1 & 0 \\
1 & -1 & 0
\end{array}\right]\left(\begin{array}{l}
v_{1} \\
v_{2} \\
v_{3}
\end{array}\right) .
\end{array}
$$

Now, integral (6.8) can be written in the form

$$
\int_{D_{1}} g_{m}^{2} \sum_{\mu=1}^{3}\left(\check{\omega}_{m} \circ \chi_{m \mu}\right)(v)\left(\check{c}_{m} \circ \chi_{m \mu}\right)(v) d v .
$$

The transform $v_{2}=\xi v_{1}$ leads to

$$
\int_{0}^{1} \int_{0}^{1} \int_{0}^{b\left(v_{1}, \xi\right)} g_{m}^{2} \sum_{\mu=1}^{3} v_{1}\left(\check{\omega}_{m} \circ \chi_{m \mu}\right)\left(v_{1}, \xi v_{1}, v_{3}\right)\left(\check{c}_{m} \circ \chi_{m \mu}\right)\left(v_{1}, \xi v_{1}, v_{3}\right) d v_{3} d \xi d v_{1} .
$$

Now, these integrals can be integrated by standard formulae. Exemplarily, we study only the case of $\mu=m=1$, where

$$
v_{1}\left(\check{\omega}_{1} \circ \chi_{11}\right)\left(v_{1}, \xi v_{1}, v_{3}\right)=\left(\frac{\xi}{(1-\xi)^{2}+\xi^{2}} \log v_{1}\right)+\left(\frac{\xi \log \sqrt{(1-\xi)^{2}+\xi^{2}}}{(1-\xi)^{2}+\xi^{2}}\right)
$$


holds. Hence, integral (6.9) can be decomposed into two integrals, where one integrand is analytic in all variables and the other is analytic in $\xi$ and $v_{3}$ and contains a logarithmic singularity of the form $\log v_{1}$. Thus, properly scaled Gauß-Legendre formulae with respect to the smooth variables and Gauß-like formulae with logarithmic weight for the integral containing $\log v_{1}$ converge exponentially.

As in Section 5 we will finish this section by explaining how the functions $c_{0}$ and $c_{1}$ appearing in (5.25) and (5.26) can be evaluated. Let a pair of points $\hat{x}, \hat{y} \in Q$ be given. First, the quantities $r=\|\hat{y}-\hat{x}\|, \xi=(\hat{y}-\hat{x}) / r, y=\eta_{i}(\hat{y}), x=\eta_{i}(\hat{x})$, and $z=y-x$ have to be computed. As in (5.24) we define

$$
c(\hat{x}, r, \xi)=r^{s} k_{i, i}(\hat{x}, r \xi+\hat{x}) .
$$

Then, $c_{0}$ is given by

$$
c_{0}(\hat{x}, \xi)=\lim _{r \rightarrow 0} c(\hat{x}, r, \xi) .
$$

We have proven that the function $c$ is analytic with respect to all variables. Hence, for small $\varepsilon>0$, the function $c_{0}(\hat{x}, \xi)$ can be approximated by

$$
c_{0}(\hat{x}, \xi)=\frac{1}{2}(c(\hat{x}, \varepsilon, \xi)+c(\hat{x},-\varepsilon, \xi))+O\left(\varepsilon^{2}\right) .
$$

For the evaluation of $c_{1}(\hat{x}, \xi)$ we use the formula

$$
c_{1}(\hat{x}, \xi)=\lim _{r \rightarrow 0} \partial_{r}(c(\hat{x}, r, \xi)) .
$$

For sufficiently small value of $\varepsilon$, this quantity can be approximated by

$$
\tilde{c}_{1}(\hat{x}, \xi)=\frac{c(\hat{x}, \varepsilon, \xi)-c(\hat{x},-\varepsilon, \xi)}{2 \varepsilon}+O\left(\varepsilon^{2}\right) .
$$

Note that these formulae are fully implicit; the derivatives of the kernel function or special expansions are never used in the cubature rules. However, if the threedimensional derivatives of the kernel function $k(x, y, z)$ are available and all second order derivatives of the chart $\eta_{i}$ as well, then $\partial_{r}(c(\hat{x}, r, \xi))$ can be expressed explicitly while the numerical evaluation might then behave more robustly with respect to cancellation errors.

It remains to explain the approximation of the function $C_{m}^{0}\left(\hat{x}_{1}\right)$ as defined in (5.35). The limit

$$
C_{m}^{0}\left(\hat{x}_{1}\right)=g_{m} \lim _{\hat{x}_{2} \rightarrow 0} \int_{a_{m-1}(\hat{x})}^{a_{m}(\hat{x})} p_{m}(\alpha) c_{0}(\hat{x}, \psi(\alpha)) d \alpha
$$

has to be computed. The integration bounds $a_{m}(\hat{x})$ can be evaluated at $\hat{x}_{2}=0$. The same holds for the function $c_{0}$. Again, the integrand is analytic and can be evaluated by properly scaled Gauß-Legendre rules.

Summarizing, we have developed cubature formulae for all integrals appearing in the context of hypersingular integral operators. First, appropriate variable transforms are applied rendering the integrand either analytic or analytic with a logarithmic weight. Such integrals finally can be treated by Gauß-like formulae. The algorithm is fully implicit, we never made use of the explicit form of the kernel function and/or the surface parametrisation. The transformations of the Gaussian points on true surface points is easy to implement and to debug since these transformations are given either by $4 \times 4$ or $3 \times 3$ matrices.

Concerning the work for assembling the system matrix, we emphasize that the number of singular cases (i.e., $\overline{K_{i}} \cap \overline{K_{j}} \neq \emptyset$ ) is proportional to $O(N)$ where $N$ 
denotes the number of panels. The integrand is regular in $O\left(N^{2}\right)$ cases. Hence, the overall complexity is dominated by the regular or so-called farfield integrals. For the singular cases, it is important to have robust cubature rules along with a proper convergence analysis to control the perturbation error arising by replacing the true Galerkin matrix by the cubature approximation (see [5]).

Alternative regularisation techniques, like partial integration or global regularisation (i.e., subtracting functions lying in the null space of the operator), have the drawback that the evaluation of the integrand for the farfield integrals is possibly more costly (cf. [10, [11) compared to the evaluation of the original kernel function. Hence, the work for approximating the farfield integrals could be substantially larger compared to using the true kernel function.

\section{ACKNOWLEDGMENTS}

It is a pleasure to acknowledge the support by the Mathematische Forschungsinstitut Oberwolfach. Most of the results of this work have been achieved during a stay of the authors at the Mathematische Forschungsinstitut. Furthermore, we would like to thank Dipl. Inform. S. Erichsen for carefully reading the manuscript and checking the formulae numerically.

\section{REFERENCES}

1. D. G. Anderson. Gaussian quadrature formula for $\int-\ln (x) f(x) d x$. Math. Comp., 19:477481, 1965. MR 31:2826

2. K. Atkinson. Solving Integral Equations on Surfaces in Space. In G. Hämmerlin and K. Hoffmann, editors, Constructive Methods for the Practical Treatment of Integral Equations, pages 20-43. Birkhäuser: ISNM, 1985. CMP 19:10

3. M. Costabel. Boundary integral operators on Lipschitz domains: Elementary results. SIAM, J. Math. Anal., 19:613-626, 1988. MR 89h:35090

4. S. Erichsen and S. Sauter. Efficient automatic quadrature in 3-d Galerkin BEM. Comp. Meth. Appl. Mech. Eng., 157:215-224, 1998. MR 99e:65163

5. I. Graham, W. Hackbusch, and S. Sauter. Discrete boundary element methods on general meshes in 3d. Technical Report 97/19, University of Bath, U.K., 1997. Mathematics Preprint, to appear in Numer. Math.

6. M. Guiggiani. Direct Evaluation of Hypersingular Integrals in 2D BEM. In W. Hackbusch, editor, Proc. Of 7th GAMM Seminar on Numerical Techniques for BEM, Kiel 1991, pages 23-34, Braunschweig, 1991. Vieweg.

7. M. Guiggiani and A. Gigante. A general algorithm for multidimensional Cauchy principal value integrals in the boundary element method. ASME J. Appl. Mech., 57:907-915. MR 93b:73037

8. W. Hackbusch. Integral Equations. ISNM. Birkhäuser, 1995. MR 96h:45001

9. W. Hackbusch and S. Sauter. On the Efficient Use of the Galerkin Method to Solve Fredholm Integral Equations. Applications of Mathematics, 38(4-5):301-322, 1993. MR 95c:65204

10. H. Han. A Boundary Element Method for Signorini Problems in Three Dimensions. Numer. Math., 60:63-76, 1991. MR 93a:65166

11. H. Han. The Boundary Integro-Differential Equations of Three-Dimensional Neumann Problem in Linear Elasticity. Numer. Math., 68(2):269-281, 1994. MR 95e:65109

12. R. Kieser. Über einseitige Sprungrelationen und hypersinguläre Operatoren in der Methode der Randelemente. PhD thesis, Mathematisches Institut A, Universität Stuttgart, Germany, 1990.

13. R. Kieser, C. Schwab, and W. L. Wendland. Numerical Evaluation of Singular and FinitePart Integrals on Curved Surfaces Using Symbolic Manipulation. Computing, 49:279-301, 1992. MR 93e:65037

14. C. Lage. Software Development for Boundary Element Mehtods: Analysis and Design of Efficient Techniques (in German). PhD thesis, Lehrstuhl Prakt. Math., Universität Kiel, 1995. 
15. J. Lyness. Applications of extrapolation techniques to multidimensional quadrature of some integrand functions with a singularity. J. Comp. Phys., 20:346-364, 1976. MR 52:15972

16. J. Lyness. An error functional expression for N-dimensional quadrature with an integrand function singular at a point. Math. Comp., 30:1-23, 1976. MR 53:11976]

17. J. Lyness and G. Monegato. Quadrature error functional expansions for the simplex when the integrand function has singularities at vertices. Math. Comp., 34:213-225, 1980. MR 80m:65017

18. S. Mikhlin and S. Prößdorf. Singular Integral Operators. Springer-Verlag, Heidelberg, 1986. MR 88e:47097

19. J. Nédélec. Curved Finite Element Methods for the Solution of Singular Integral Equations on Surfaces in $\mathbf{R}^{3}$. Comput. Meth. Appl. Mech. Engrg., 8:61-80, 1976. MR 58:19275

20. J. Nédélec. Integral Equations with Non Integrable Kernels. Integral Equations Oper. Theory, 5:562-572, 1982. MR 84i:45011

21. O. Oleinik, A. Shamaev, and G. Yosifian. Mathematical Problems in Elasticity and Homogenization. North-Holland, Amsterdam, 1992. MR 93k:35025

22. S. Sauter and C. Lage. Transformation of hypersingular integrals and black-box cubature (extended version). Technical Report 97-17, Universität Kiel, 1997. (Available via WWWaddress: http://www.numerik.uni-kiel.de/reports/1997/).

23. S. A. Sauter. Über die effiziente Verwendung des Galerkinverfahrens zur Lösung Fredholmscher Integralgleichungen. PhD thesis, Inst. f. Prakt. Math., Universität Kiel, 1992.

24. S. A. Sauter and A. Krapp. On the Effect of Numerical Integration in the Galerkin Boundary Element Method. Numer. Math., 74(3):337-360, 1996. MR 97j:65189

25. C. Schwab and W. Wendland. Kernel Properties and Representations of Boundary Integral Operators. Math. Nachr., 156:187-218, 1992. MR 94g:65135

26. C. Schwab and W. Wendland. On Numerical Cubatures of Singular Surface Integrals in Boundary Element Methods. Numer. Math., 62:343-369, 1992. MR 93h:65035

27. T.von Petersdorff and C. Schwab. Fully Discrete Multiscale Galerkin BEM. In W. Dahmen, P. Kurdila, and P. Oswald, editors, Multiresolution Analysis and Partial Differential Equations, pages 287-346, New York, 1997. Academic Press. MR 99a:65158

28. W. Wendland. Strongly elliptic boundary integral equations. In A. Iserles and M. Powell, editors, The State of the Art in Numerical Analysis, pages 511-561, Oxford, 1987. Clarendon Press. MR 88m:65209

Institut FÜr Mathematik, Universität Zürich, Winterthurerstr. 190, CH-8050 Zürich, SWITZERLAND

E-mail address: stas@amath.unizh.ch

Coyote Systems, 2740 Van Ness Avenue \#210, San Francisco, CA 94109

E-mail address: lage@coyotesystems.com 